\title{
Pressure Decimation and Interpolation (PDI) method for a Baroclinic Non-hydrostatic Model
}

\author{
Jian Shi ${ }^{1,2}$, Fengyan $\mathrm{Shi}^{2}$, James T. Kirby ${ }^{2}$, Gangfeng $\mathrm{Ma}^{3}$, Guoxiang $\mathrm{Wu}^{2,4}$ \\ , Chaofeng Tong ${ }^{1}$, Jinhai Zheng ${ }^{1}$ \\ ${ }^{1}$ College of Harbor, Coastal and Offshore Engineering, Hohai University, Nanjing, \\ 210098, China \\ ${ }^{2}$ Center for Applied Coastal Research, Department of Civil and Environmental \\ Engineering, University of Delaware, Newark, DE 19716, USA \\ ${ }^{3}$ Department of Civil and Environmental Engineering, Old Dominion University, \\ Norfolk, VA 23529, USA \\ ${ }^{4}$ College of Engineering, Ocean University of China, Qingdao, 266100, China \\ Corresponding author: Tel: 86-13770984093; Email:address: Jianshi@udel.edu
}

\begin{abstract}
Non-hydrostatic models are computationally expensive in simulating density flows and mass transport problems due to the requirement of sufficient grid resolution to resolve density and flow structures. Numerical tests based on the Non-Hydrostatic Wave Model, NHWAVE [Ma et al., 2012], indicated that up to $70 \%$ of the total computational cost may be born by the pressure Poisson solver in cases with high grid resolution in both vertical and horizontal directions. However, recent studies using Poisson solver-based non-hydrostatic models have shown that an accurate prediction of wave dispersion does not require a large number of vertical layers if the dynamic pressure is properly discretized. In this study, we explore the possibility that the solution for the dynamic pressure field may, in general, be decimated to
\end{abstract}


a resolution far coarser than that used in representing velocities and other transported quantities, without sacrificing accuracy of solutions. Following van Reeuwijk [2002], we determine the dynamic pressure field by solving the Poisson equation on a coarser grid and then interpolate the pressure field onto a finer grid used for solving for the remaining dynamic variables. With the Pressure Decimation and Interpolation (PDI) method, computational efficiency is greatly improved. We use three test cases to demonstrate the model's accuracy and efficiency in modeling density flows.

Keywords:

Non-hydrostatic wave model, Pressure Decimation and Interpolation, Baroclinic model

1. Introduction

2

Since the late 1990s, with the rapid growth of computing power, many 3 efforts have been made to develop non-hydrostatic models for applications 4 to modeling surface waves, internal waves and tides, and nearshore density 5 flows. In contrast to models based on the hydrostatic pressure assumption, 6 non-hydrostatic models are also valid for simulating flows in which the effects 7 of vertical acceleration are not negligible, such as dispersive waves, strongly stratified fluids and flows over rapid bed slope changes. In the nearshore research community, several non-hydrostatic constant-density models based on vertical $\sigma$-coordinates have been developed for modeling surface wave trans11 formation from deep water to the swash zone [Yuan and Wu, 2004; Zijlema 
et al., 2011, Bradford 2011, Ma et al., 2012]. These models are attractive due to their computational efficiency in modeling waves in a large computational domain, compared to traditional Navier-Stokes solvers using surface tracking methods such as the volume-of-fluid (VOF) [Hirt and Nichols, 1981] or the marker-and-cell (MAC) [Harlow and Welch, 1965] methods. In addition, the models are conceptually simple in comparison to the Boussinesq class of models for dispersive waves [Shi et al., 2012] and their further extensions to incorporate turbulence and stratification effects [Kim et al., 2009, Kim and Lynett, 2011].

As in traditional Navier-Stokes solvers, the two-step projection method (or the pressure-correcting projection method, Casulli, 1999) is usually used in solving the $\sigma$-coordinate based equations. The total pressure is divided into two parts: dynamic pressure and hydrostatic pressure. In the first step, intermediate values of velocities are computed from the momentum equations with the dynamic pressure term ignored. In the second step, the dynamic pressure is determined by solving the pressure Poisson equation, which is obtained by coupling the continuity equation and momentum equations forced by the dynamic pressure only. An alternative approach to implement the two-step projection method is that the intermediate values of velocities are estimated using the dynamic pressure at the previous time level in the first step. In the second step, the Poisson equation obtained in the same way as in the first approach becomes the equation with respect to the dynamic pressure increment (in time) instead of the dynamic pressure itself (Goda, 1979, van 
Kan, 1986 and Guermond et al., 2006). In both approaches, the velocity fields are corrected using the updated dynamic pressure. The solution of the pressure Poisson equation is the most time consuming part of the numerical scheme; our own tests based on the Non-Hydrostatic Wave Model NHWAVE [Ma et al., 2012] also indicated that up to $70 \%$ of the computational cost resides in the pressure Poisson solver in cases with high grid resolution.

Recent studies also show that the numerical scheme for solving the dynamic pressure is crucial for model accuracy and efficiency. The NavierStokes solvers based on a staggered grid framework in which the pressure is set in the grid cell center, e.g., Mahadevan et al. [1996], Namin et al. [2001] and Chen [2003], have difficulties in accurately implementing the boundary condition for the pressure at the free surface, and thus require a considerable number of vertical layers (10-20) to get accurate wave dispersion characteristics. Stelling and Zijlema [2003] proposed an approximation of the vertical gradient of the non-hydrostatic pressure based on the Kellerbox scheme, which locates the pressure at the upper cell faces so that the dynamic pressure at the water surface can be exactly set to zero without any approximation. Ma et al., [2012] followed the idea of the Keller-box scheme to locate the dynamic pressure at the cell faces at which the velocity values are estimated by interpolating the cell-centered values to the cell faces when constructing the pressure Poisson equation. Young and $\mathrm{Wu}[2009,2010]$ used a non-hydrostatic pressure distribution at the top layer provided by Boussinesq equations to optimize wave dispersion property. The models using either 
Keller-box scheme or Boussinesq type scheme can efficiently and accurately simulate highly dispersive waves with a very small number (3-5) of vertical layers, indicating a weak dependence of the wave dispersion prediction on the vertical resolution of the model.

For modeling stratified flows and mass transport problems, a high vertical resolution for velocity and density fields is needed. To improve computing efficiency, the traditional shallow water hydrostatic models, such as ROMS [Shchepetkin and McWilliams, 2005], Delft3D [Stelling, 1984, 1986], FVCOM [Chen et al., 2003], GETM [Burchard and Bolding, 2002] and HHU-ECR3D [Zheng et al., 2002] use mode splitting schemes. The models are separated into two components: external mode and internal mode. In the external mode, the governing equations are the depth-integrated continuity and momentum equations, which are less expensive to be solved. However, as a requirement for simulating gravity waves, the external mode time step is limited by the Courant-Friedrichs-Lewy condition. The baroclinic equations govern the internal mode, which are more expensive to be solved but can be solved at much larger time steps due to the smaller celerity of internal waves.

An ocean model with the hydrostatic pressure assumption is not able to predict non-hydrostatic effects, as significant vertical velocity on small scales is present. Vertical velocity and acceleration may be large in dispersive waves, strongly stratified fluids, turbulent fluctuations, and flows over a rapidly varying bottom topography. Therefore, many efforts have been made to extend the hydrostatic ocean models into non-hydrostatic solvers [Jankowski, 
1999, Kanarska and Maderich, 2003, Heggelund et al., 2004, Berntsen and Furnes, 2005, Kanarska et al., 2007, Lai et al., 2010a, b, and Auclair et al., 2011] for applications of non-hydrostatic processes. Those extensions were usually implemented using the pressure-correcting projection method, which involves the time consuming pressure Poisson solver.

An alternative approach to include the non-hydrostatic pressure contribution into an existing hydrostatic model can be made based on the direct implementation of the non-hydrostatic pressure which is integrated from the vertical momentum equation into the horizontal pressure gradient terms in the horizontal momentum equations. This procedure is explicit and decoupled between the mass conservation equation and the momentum equations. The approach is suitable for weakly non-hydrostatic flows, assuming that the dominant part of the pressure field is hydrostatic and the non-hydrostatic effects represent a small correction. This straight-forward inclusion of the nonhydrostatic pressure contribution into a hydrostatic model does not require the inversion of a Poisson equation and have been applied to several modeling systems such as in Dietrich and Lin [2002], Johns [1991], Johns and Xing [1993], Li and Johns [2001], and Gaarthuis [1995]. Recently, Klingbeil and Burchard [2013] carried out a validation of the non-hydrostatic capability of the straight-forward method used in the full 3D mode-splitting model GETM. They pointed out that the treatment of the incompressibility constraint and the balance of momentum in a decoupled manner strongly affected the model stability. A cumulative temporal filter was suggested to be used in the 
solver to suppress instabilities. In addition, the coupling convergence could be improved by a high number of iterations which could strongly hamper the model efficiency. It was concluded in Klingbeil and Burchard [2013] that the straight-forward inclusion of non-hydrostatic dynamics cannot replace the pressure correcting projection method but can be used in the limit of weakly non-hydrostatic processes.

Many efforts have been conducted to improve the efficiency of non-hydrostatic models by simplifying the Poisson equation. Berntsen and Furnes [2005] obtained a simpler set of Poisson equation by modelling the non-hydrostatic pressure in the $\sigma$-coordinates. This method is an approximation to the Poisson equation and the errors increase with the steepness of the topography(Keilegavlen and Berntsen, 2009). Scotti and Mitran [2008] approximated the solution of the pressure Poisson equation by at most three terms of an expansion in powers of the ratio $\Delta x / H$. This method is valid for simulating nonlinear internal waves for grids with the ratio less than 2. Cui [2013] and Cui et al. [2014] reduced the size of the Poisson equation by using a parameterized non-hydrostatic pressure distribution in the bottom cell. The benefit of this method is significant in two layer models but the model is less efficien$\mathrm{t}$ as a larger number of vertical layers is used. Vitousek and Fringer [2014] developed a non-hydrostatic, isopycnal-coordinate ocean model, which is efficient for reducing the number of vertical layers, but the isopycnal-coordinate system can be invalid for modeling over-turning processes such as internal wave breaking and Kelvin-Helmholtz instabilities. 
The motivation of this study is to find an efficient method for modeling dispersive waves in a stratified environment using a non-hydrostatic model. As mentioned above, a higher vertical resolution may be needed for simulations involving baroclinic forcing and mass transport, while an accurate prediction of dynamic pressure field may not require a large number of vertical layers. It is expected that a reduction of the number of vertical layers in solving the dynamic pressure can significantly improve the computational efficiency of a non-hydrostatic model. van Reeuwijk [2002] has used the strategy to develop a non-hydrostatic 2DV free surface wave model. In van Reeuwijk, independent numbers of pressure layers and horizontal velocity layers were implemented with a smaller number of pressure layers than that of velocity layers. An extensive investigation was carried out to examine the interpolation method which was used to re-distribute the dynamic pressure field from the pressure points into the velocity points. The model with fewer pressure layers than velocity layers showed a greater efficiency in modeling short wave propagation without losing much accuracy.

In this study, we focus on stratified flow problems with the wave dispersion characteristics. Our hypothesis is that an accurate prediction of the dynamic pressure field may not require a high resolution grid, especially in the vertical direction. The solution for the dynamic pressure can be decimated by solving the Poisson equation with a coarser grid compared to the regular grid used for solving the rest of the equations for velocity, turbulence and passive tracers. Then, an interpolation can be performed to specify the pressure field on the 
finer grid used for solving the remaining equations. A coarser grid is used in solving the pressure Poisson equation and a finer grid is used for the velocity, turbulence and equations for passive tracer components such as salinity and temperature. Numerical tests with different grid configurations are carried out against theoretical solutions or measured data. The PDI method is also used in the horizontal direction to examine if the model efficiency can be further improved.

The paper is organized as follows. The model formulation is summarized in section 2, followed by the introduction to the PDI algorithm. In section 3, three numerical experiments, the lock exchange problem, internal wave breaking and a two-layer flow over a fixed sill, were carried out to demonstrate the capability of PDI in simulating density flows. Section 4 is devoted to a discussion of the accuracy and computational efficiency of the model. Finally, conclusions are drawn in section 5 .

\section{Model description}

\subsection{Governing equations}

The complete NHWAVE equations, describing non-hydrostatic motion, turbulent mixing, baroclinic forcing and mass transport, can be found in Ma et al. [2013]. Here, we only list the equations related to the PDI method in the present study.

The generalized conservative form of mass and momentum equations can 
171 be written as

$$
\frac{\partial \Psi}{\partial t}+\nabla \cdot \boldsymbol{\Theta}(\Psi)=\mathbf{S}
$$

172 where $\nabla=\left(\frac{\partial}{\partial x}, \frac{\partial}{\partial y}, \frac{\partial}{\partial \sigma}\right)$. $\Psi$ and $\boldsymbol{\Theta}(\Psi)$ are the vector of conserved variables 173 and the flux vector respectively.

$$
\begin{gathered}
\Psi=\left(\begin{array}{c}
D \\
D u \\
D v \\
D w
\end{array}\right) \\
\Theta=\left(\begin{array}{c}
\left(D u u+\left(\frac{1}{2} g \eta^{2}+g h \eta\right)\right) \mathbf{i}+D u v \mathbf{j}+u \omega \mathbf{k} \\
D u v \mathbf{i}+\left(D v v+\left(\frac{1}{2} g \eta^{2}+g h \eta\right)\right) \mathbf{j}+v \omega \mathbf{k} \\
D u w \mathbf{i}+D v w \mathbf{j}+w \omega \mathbf{k}
\end{array}\right) .
\end{gathered}
$$

174 where $D=h+\eta, h$ is water depth and $\eta$ is surface elevation, $(u, v, w)$ 175 represent velocity components in the Cartesian coordinate system $\left(x^{*}, y^{*}, z^{*}\right)$ 176 and $\omega$ is the velocity normal to the $\sigma$ levels. The source term on the right 177 hand side includes several source components:

$$
\mathbf{S}=\mathbf{S}_{h}+\mathbf{S}_{p}+\mathbf{S}_{\rho}+\mathbf{S}_{\tau}
$$


where $\mathbf{S}_{h}, \mathbf{S}_{p}, \mathbf{S}_{\rho}$ and $\mathbf{S}_{\tau}$ represent the bottom slope term, dynamic pressure gradient, baroclinic forcing and turbulent mixing respectively. These terms are expressed as below.

$$
\begin{gathered}
\mathbf{S}_{h}=\left(\begin{array}{c}
g \eta \frac{\partial h}{\partial x} \\
g \eta \frac{\partial h}{\partial y} \\
0
\end{array}\right) \quad \mathbf{S}_{p}=\left(\begin{array}{c}
-\frac{D}{\rho}\left(\frac{\partial p}{\partial x}+\frac{\partial p}{\partial \sigma} \frac{\partial \sigma}{\partial x^{*}}\right) \\
-\frac{D}{\rho}\left(\frac{\partial p}{\partial y}+\frac{\partial p}{\partial \sigma} \frac{\partial \sigma}{\partial y^{*}}\right) \\
-\frac{1}{\rho} \frac{\partial p}{\partial \sigma}
\end{array}\right) \\
\mathbf{S}_{\rho}=\left(\begin{array}{c}
-g D\left(\frac{\partial r}{\partial x}+\frac{\partial r}{\partial \sigma} \frac{\partial \sigma}{\partial x^{*}}\right) \\
-g D\left(\frac{\partial r}{\partial y}+\frac{\partial r}{\partial \sigma} \frac{\partial \sigma}{\partial y^{*}}\right) \\
-g \frac{\partial r}{\partial \sigma}
\end{array}\right) \mathbf{S}_{\tau}=\left(\begin{array}{c}
D S_{\tau x} \\
D S_{\tau y} \\
D S_{\tau z}
\end{array}\right)
\end{gathered}
$$

where $p$ represents the dynamic pressure and $r$ is the baroclinic pressure head calculated by

$$
r=\frac{D}{\rho_{0}} \int_{\sigma}^{1} \rho d \sigma
$$

where $\rho_{0}$ is the reference density and $\rho$ is the dynamic density defined in Ma et al. [2013]. The turbulent diffusion term $S_{\tau x}, S_{\tau y}$ and $S_{\tau z}$ are given by

$$
\begin{aligned}
& S_{\tau x}=\frac{\partial \tau_{x x}}{\partial x}+\frac{\partial \tau_{x x}}{\partial \sigma} \frac{\partial \sigma}{\partial x^{*}}+\frac{\partial \tau_{x y}}{\partial y}+\frac{\partial \tau_{x y}}{\partial \sigma} \frac{\partial \sigma}{\partial y^{*}}+\frac{\partial \tau_{x z}}{\partial \sigma} \frac{\partial \sigma}{\partial z^{*}} \\
& S_{\tau y}=\frac{\partial \tau_{y x}}{\partial x}+\frac{\partial \tau_{y x}}{\partial \sigma} \frac{\partial \sigma}{\partial x^{*}}+\frac{\partial \tau_{y y}}{\partial y}+\frac{\partial \tau_{y y}}{\partial \sigma} \frac{\partial \sigma}{\partial y^{*}}+\frac{\partial \tau_{y z}}{\partial \sigma} \frac{\partial \sigma}{\partial z^{*}} \\
& S_{\tau z}=\frac{\partial \tau_{z x}}{\partial x}+\frac{\partial \tau_{z x}}{\partial \sigma} \frac{\partial \sigma}{\partial x^{*}}+\frac{\partial \tau_{z y}}{\partial y}+\frac{\partial \tau_{z y}}{\partial \sigma} \frac{\partial \sigma}{\partial y^{*}}+\frac{\partial \tau_{z z}}{\partial \sigma} \frac{\partial \sigma}{\partial z^{*}}
\end{aligned}
$$


186

calculated as

$$
\begin{aligned}
& \tau_{x x}=2 \nu_{t}\left(\frac{\partial u}{\partial x}+\frac{\partial u}{\partial \sigma} \frac{\partial \sigma}{\partial x^{*}}\right) \quad \tau_{y y}=2 \nu_{t}\left(\frac{\partial v}{\partial y}+\frac{\partial v}{\partial \sigma} \frac{\partial \sigma}{\partial y^{*}}\right) \quad \tau_{z z}=2 \nu_{t}\left(\frac{\partial w}{\partial \sigma} \frac{\partial \sigma}{\partial z^{*}}\right) \\
& \tau_{x y}=\tau_{y x}=\nu_{t}\left(\frac{\partial u}{\partial y}+\frac{\partial u}{\partial \sigma} \frac{\partial \sigma}{\partial y^{*}}+\frac{\partial v}{\partial x}+\frac{\partial v}{\partial \sigma} \frac{\partial \sigma}{\partial x^{*}}\right) \\
& \tau_{x z}=\tau_{z x}=\nu_{t}\left(\frac{\partial u}{\partial \sigma} \frac{\partial \sigma}{\partial z^{*}}+\frac{\partial w}{\partial x}+\frac{\partial w}{\partial \sigma} \frac{\partial \sigma}{\partial x^{*}}\right) \\
& \tau_{y z}=\tau_{z y}=\nu_{t}\left(\frac{\partial v}{\partial \sigma} \frac{\partial \sigma}{\partial z^{*}}+\frac{\partial w}{\partial y}+\frac{\partial w}{\partial \sigma} \frac{\partial \sigma}{\partial y^{*}}\right)
\end{aligned}
$$

where the $\nu_{t}$ is the turbulent kinematic viscosity. In the first two cases in Section 3 , the values of viscosity $\nu_{t}$ are set to be constant as described in the specific cases. In the last case, the model is run using a LES approach (Pope, 2000) and the standard Smagorinsky (1963) model for turbulent viscosity given by

$$
\nu_{t}=\left(C_{s} \delta\right)^{2} \sqrt{2 S_{i j} S_{i j}}
$$

where $C_{s}$ is the Smagorinsky coefficient, $\delta=(\Delta x \Delta y \Delta z)^{1 / 3}$ and $S_{i j}=\frac{1}{2}\left(\frac{\partial u_{i}}{\partial x_{j}^{*}}+\right.$ $\left.\frac{\partial u_{j}}{\partial x_{i}^{*}}\right)$ is the stress tensor. The value of the $C_{s}$ is taken as 0.15 in the last test case.

The governing equation for free surface can be written as

$$
\frac{\partial D}{\partial t}+\frac{\partial}{\partial x}\left(D \int_{0}^{1} u d \sigma\right)+\frac{\partial}{\partial y}\left(D \int_{0}^{1} v d \sigma\right)=0
$$

The advection and diffusion equation governing the motion of passive tracers can be expressed in the similar conservative form as in (1)

$$
\frac{\partial \boldsymbol{\Psi}_{c}}{\partial t}+\nabla \cdot \boldsymbol{\Theta}_{c}\left(\boldsymbol{\Psi}_{c}\right)=\mathbf{S}_{c}
$$


where $\boldsymbol{\Psi}_{c}$ is a passive tracer scaler such as water temperature, salinity, turbulent kinetic energy and dissipation, etc. $\mathbf{S}_{c}$ represents the source/sink term. Here, $\Psi_{c}$ is a concentration variable $C$ of a passive tracer; then

$$
\Theta_{c}(C)=(D u C \mathbf{i}+D v C \mathbf{j}+\omega C \mathbf{k})
$$

Numerically, NHWAVE uses a combined finite-volume and finite-difference scheme with a Godunov-type method for the spatial discretization and a twostage (second-order) SSP Runge-Kutta (R-K) scheme [Gottlieb et al., 2001] for time stepping. The two-step projection method, which splits the time integration into a hydrostatic predictor phase and the non-hydrostatic corrector phase, is used within each R-K stage. Here, we only illustrate the first stage of R-K for brevity.

In the hydrostatic phase, an intermediate quantity of $\mathbf{U}$, where $\mathbf{U}=$ $(D u, D v, D w)^{T}$, is evaluated using the momentum equations with the dynamic pressure term neglected:

$$
\frac{\mathbf{U}^{*}-\mathbf{U}^{n}}{\Delta t}=-\nabla \cdot \boldsymbol{\Theta}(\mathbf{U})^{n}+\mathbf{S}_{h}^{n}+\mathbf{S}_{\rho}^{n}+\mathbf{S}_{\tau}^{n}
$$

where $\mathbf{U}^{n}$ represents $\mathbf{U}$ value at time level $n$ and $\mathbf{U}^{*}$ is the intermediate value from the hydrostatic phase. The fluxes in Eq. (13) are discretized using a second-order Godunov-type finite volume method. In the present version of NHWAVE, the Superbee (Roe, 1986) and van Leer (van Leer, 1974) limiters are implemented. Our tests show that the van Leer limiter is more dissipative 
than the Superbee limiter and thus the Superbee limiter is used for all test cases in the present study.

In the non-hydrostatic phase, the velocity field is corrected using the dynamic pressure term:

$$
\frac{\mathbf{U}^{(1)}-U^{*}}{\Delta t}=\mathbf{S}_{p}^{(1)}
$$

where the superscription ()$^{(1)}$ represents the first stage for the R-K scheme. Substituting (14) into the continuity equation

$$
\frac{\partial u}{\partial x}+\frac{\partial u}{\partial \sigma} \frac{\partial \sigma}{\partial x^{*}}+\frac{\partial v}{\partial y}+\frac{\partial v}{\partial \sigma} \frac{\partial \sigma}{\partial y^{*}}+\frac{1}{D} \frac{\partial w}{\partial \sigma}=0
$$

yields the Poisson equation of the dynamic pressure

$$
\begin{aligned}
& \frac{\partial}{\partial x}\left[\frac{\partial p}{\partial x}+\frac{\partial p}{\partial \sigma} \frac{\partial \sigma}{\partial x^{*}}\right]+\frac{\partial}{\partial y}\left[\frac{\partial p}{\partial y}+\frac{\partial p}{\partial \sigma} \frac{\partial \sigma}{\partial y^{*}}\right]+\frac{\partial}{\partial \sigma}\left(\frac{\partial p}{\partial x}\right) \frac{\partial \sigma}{\partial x^{*}}+ \\
& \frac{\partial}{\partial \sigma}\left(\frac{\partial p}{\partial y}\right) \frac{\partial \sigma}{\partial y^{*}}+\left[\left(\frac{\partial \sigma}{\partial x^{*}}\right)^{2}+\left(\frac{\partial \sigma}{\partial y^{*}}\right)^{2}+\frac{1}{D^{2}}\right] \frac{\partial}{\partial \sigma}\left(\frac{\partial p}{\partial \sigma}\right)= \\
& \frac{\rho}{\Delta t}\left(\frac{\partial u^{*}}{\partial x}+\frac{\partial u^{*}}{\partial \sigma} \frac{\partial \sigma}{\partial x^{*}}+\frac{\partial v^{*}}{\partial y}+\frac{\partial v^{*}}{\partial \sigma} \frac{\partial \sigma}{\partial y^{*}}+\frac{1}{D} \frac{\partial w^{*}}{\partial \sigma}\right)
\end{aligned}
$$

23. The same procedure is used for the second stage of the R-K scheme. The transport of a passive tracer is calculated using (11) with the velocity field corrected in the non-hydrostatic phase.

For the computational grid arrangement, the points for velocity and tracer variables are defined at the cell centers. The dynamic pressure for the Keller-box scheme is defined at the cell faces. The velocity values at the 
cell faces, $\left(u^{*}, v^{*}, w^{*}\right)$, are obtained by linear interpolation using the adjacent cell-centered values for the discretization of the Poisson equation (16).

\subsection{Pressure decimation and interpolation (PDI)}

The PDI method is implemented by choosing a subset of vertical grid points from the full velocity grid and implements the Poisson solver for dynamic pressure only on the subset. Fig. 1 demonstrates the grid arrangement in the vertical direction with a coarse grid with less vertical layers shown in solid lines and a fine grid with more vertical layers in dashed lines. The same grid arrangement is done in the horizontal directions. In the coarse grid, the dynamic pressure $\left(p^{\prime}\right)$ points are located at the cell faces and velocity points $\left(u^{\prime}, w^{\prime}\right)$ at the cell centers. The same configuration is made for the fine grid and the corresponding variables are denoted by $(p, u, w)$ in the figure. $(u, w)$ and $\left(u^{\prime}, w^{\prime}\right)$ are overlapped at the cell center of the coarse grid, which is unnecessary. It should be mentioned that, the pressure points in the coarse grid are uniformly distributed in the $\sigma$ coordinate, in contrast to van Reeuwijk who applied a non-uniform distribution with more pressure points at surface and less pressure points at the bottom for unstratified surface wave simulations.

Fig. 2 illustrates the flow chart of PDI. The variables in a coarse grid and in a fine grid are solved alternatively in the time integration loops. Detailed procedures are listed as follows, and again, only the first stage of R-K scheme is shown for demonstration. 
Step 1: the intermediate values $\mathbf{U}^{*}$ is evaluated on the fine grid by solving (13).

Step 2: $\mathbf{U}^{*^{\prime}}$ on the coarse grid is obtained by piecewise-averaging $\mathbf{U}^{*}$ from the fine grid. The piecewise-averaging method can be given by:

$$
\mathbf{U}_{\mathbf{i}}^{*^{\prime}}=\sum_{j=J_{i}}^{J_{i}+N_{i}-1} \frac{\mathbf{U}_{j}^{*}}{N_{i}}
$$

Step 3: the dynamic pressure $p^{\prime}$ is calculated on the coarse grid by solving (16).

Step 4: the dynamic pressure $p$ on the fine grid is obtained using an interpolation method.

Step 5: the velocity field is corrected on the fine grid using the equation (14).

Step 6: free surface positions are obtained using equation (10). Equation (11) for the passive tracers are solved on the fine grid. The turbulent eddy viscosity and baroclinic forcing are calculated and used for the next time step on the fine grid.

Note that either $\mathbf{U}^{*}$ on the fine grid or $\mathbf{U}^{*^{\prime}}$ on the coarse grid does not 
guarantee mass or volume conservation. However, the incompressible condition in the coarse grid is satisfied by using (16) in Step 3, while the mass conservation in the fine grid is guaranteed by using the vertically integrated continuity equation (10).

Different interpolation methods, including the linear, quadratic, cubic spine interpolation and the fourth-order spline interpolation [Van Reeuwijk, 2002], were tested. The cubic spline interpolation gives the most accurate estimation. So we only described the detail of the cubic spline interpolation. A cubic spline is a spline constructed of piecewise third-order polynomials which can be expressed as

$$
p_{i}(\sigma)=a_{i}\left(\sigma-\sigma_{i}\right)^{3}+b_{i}\left(\sigma-\sigma_{i}\right)^{2}+c_{i}\left(\sigma-\sigma_{i}\right)+d_{i}
$$

where $p_{i}(\sigma)$ is the cubic polynomial of the dynamic pressure in each interval. $a_{i}, b_{i}, c_{i}$ and $d_{i}$ are the coefficients. $i$ is the index on the coarse grid.

We define $n_{u}$ as the number of vertical grid cells for velocity calculation and $n_{p}$ as the number of vertical grid cells for pressure calculation. There are $n_{p}+1$ control points and $n_{p}$ intervals, which means there are $4 n_{p}$ independent conditions needed to fix the spline. We assume that the cubic polynomials match the values at both ends of the intervals and the first and second derivatives are continuous, which can provides $4 n_{p}-2$ conditions. Two extra conditions are needed. The following boundary conditions of the dynamic 
288

pressure at the bottom and surface are applied:

$$
\begin{aligned}
\frac{\partial p}{\partial \sigma} & =0 ; & & \sigma=0 \\
\frac{\partial^{2} p}{\partial \sigma^{2}} & =0 ; & & \sigma=1
\end{aligned}
$$

\section{Numerical experiments}

We conducted three numerical experiments to test the PDI method in simulating density flows. The first case is the lock exchange problem, which demonstrates the efficiency and accuracy of PDI in simulating the extreme baroclinic dynamics. The second case is a simulation of internal wave breaking, which is compared to the laboratory experiment data. The third case is a simulation of two-layer flow over a fixed sill. The first two cases are carried out in two dimensions, with the domain in a vertical plane. The third case is three dimensional and utilized to explore the benefits of the PDI method in a 3D application. In this section, we focused on tests of PDI in the vertical direction only. The applications of the PDI in the horizontal direction appeared to be less promising than the vertical PDI in terms of model efficiency improvement. The PDI method in the horizontal direction will be discussed in Section 4.

\subsection{The lock-exchange problem}

The lock exchange problem, which is the gravitational adjustment of two fluids of different density initially separated by a vertical gate, has become 
a standard case for testing the baroclinic dynamics component of numerical models [Bourgault and Kelley, 2004; Fringer et al., 2006; Lai et al., 2010a; Ma et al., 2013]. The density is assumed to be expressed as follows:

$$
\rho=999.972 \times\left(1+0.75 \times 10^{-3} S\right)
$$

The light fluid is set to be freshwater $\left(\rho_{1}=999.972 \mathrm{~kg} / \mathrm{m}^{3}\right)$ with zero salinity and the heavy fluid $\left(\rho_{2}=1000.991 \mathrm{~kg} / \mathrm{m}^{3}\right)$ is set to be salt water with a salinity of $1.3592 \mathrm{psu}$. The two kinds of fluids are separated by a gate in the middle of the domain. The salinity is defined as:

$$
S(x, z)=\left\{\begin{array}{cl}
1.3592 & x \geq 0.4 \mathrm{~m} \\
0 & x<0.4 \mathrm{~m}
\end{array}\right.
$$

The numerical domain is a $2 \mathrm{D}$ flume with a length $(L=0.8 \mathrm{~m})$ and a static water depth $(H=0.1 \mathrm{~m})$. The horizonal resolution is $\Delta x=0.001$ m. Five cases with, respectively, $(200,200)\left(\left(n_{u}, n_{p}\right)\right.$, thereafter $),(200,20)$, $(100,20),(50,20)$ and $(20,20)$ vertical layers were conducted to investigate the influence of PDI on the model accuracy and efficiency. The molecular viscosity is $10^{-6} \mathrm{~m}^{2} / \mathrm{s}$. The eddy viscosities are set to be zero. The slope limiter used in the shock-capturing scheme is the Superbee limiter.

After the gate between the fresh water and the heavy fluid is removed at $t=0 \mathrm{~s}$, a gravitational current develops between the interface of the two fluids due to the baroclinic forcing. With an increase of velocity shear, the 
Kelvin-Helmholtz billows arise across the interface. Fig. 3(a)-(c) compare the snapshots of density distribution at $t=20 \mathrm{~s}$ between the cases with $(20,20),(200,20)$ and $(200,200)$ vertical layers. The results of $(20,20)$ shows that the vorticity cores are not well resolved at the low grid resolution, while the comparison between the cases of $(200,20)$ and $(200,200)$ does not show an obvious difference. It indicates that the PDI model with less pressure layers can successfully predict salinity and velocity fields. Fig. 3(d) shows the snapshot of the hydrostatic simulation. The billows cannot be predicted by the model without dynamic pressure, indicating that the dynamic pressure component plays an important role in the generation and evolution of the Kelvin-Helmholtz billows. The comparisons of the horizontal velocities at the midpoint of the computational domain between the model simulations and the theoretical value $(1.59 \mathrm{~cm} / \mathrm{s})$ derived by Jankowski [1999] are presented in Fig. 4. The maximum velocities at the surface and bottom are very close to the theoretical values. The model slightly underestimates the terminal velocity from $t=17.0 \mathrm{~s}$, which may be induced by the influence of the left and right boundaries. As the case is simulated in a finite domain, the velocities cannot reach an asymptotic value.

The vertical distributions of velocity $u$ and $w$, salinity $S$ and dynamic pressure $p$ predicted from models with the grid configurations of $(200,200)$, $(100,20),(50,20)$ and $(20,20)$, at $t=20 \mathrm{~s}$, are compared at two sections as shown in Figs. 5 and 6 . One of the sections is located between two vortex cells at $x=0.31 \mathrm{~m}$, and the other is located across a vortex cell 
at $x=0.19 \mathrm{~m}$. The comparisons show that the results of $(200,20)$ layers agree well with the results of $(200,200)$ layers for all compared variables (black lines for $(200,200)$ and red lines for $(200,20)$ are indistinguishable). There are not much differences in $u$ between the cases of $(200,200),(100,20)$, $(50,20)$, except for the case of $(20,20)$ as shown in the left panels of Fig. 5. In the comparisons of the vertical distribution of salinity, large discrepancies can be found at the selected sections (right panels of Fig. 5) between the case of $(20,20)$ and other cases. The large discrepancies are caused by a big difference in the vortex core locations predicted by the $(20,20)$ model and other models. More significant differences between the results from the full grid model $((200,200)$, thereafter $)$ and the PDI models can be found in the comparisons of $w$ and $p$ as shown in Fig. 6. Except the case of $(200,20), w$ and $p$ predicted by the PDI models apparently deviate from those predicted by the full grid model. The deviation increases with a decrease in the velocity grid resolution. The case of the $(20,20)$ grid configuration shows the largest pressure discrepancy from the full grid model, especially at $x=0.31 \mathrm{~m}$, where the opposite pressure values were predicted compared to the full grid model.

Tests on further reducing the number of pressure layers to less than 20 did not show a successful generation of the Kelvin-Helmholtz billows, probably because the low vertical resolution could not resolve the vertical structure of dynamic pressure in the lock exchange case.

To investigate why the non-hydrostatic pressure field can be decimat- 
ed, but not the velocity/tracer field, in modeling non-hydrostatic, baroclinic flows, we examined the vorticity generation mechanism in the lock exchange problem. The vorticity conservation equation for the lock exchange problem can be written as

$$
\begin{aligned}
\frac{d \boldsymbol{\Omega}}{d t} & =(\boldsymbol{\Omega} \cdot \nabla) \mathbf{U} \\
& +\frac{1}{\rho^{2}}\left(\nabla \rho \times \nabla p_{\mathrm{S}}+\nabla \rho \times \nabla p_{\mathrm{nh}}\right) \\
& +\nabla \times\left(\frac{\nabla \cdot \tau}{\rho}\right)
\end{aligned}
$$

where $\Omega$ represents the vorticity vector. $p_{\mathrm{S}}$ and $p_{\mathrm{nh}}$ represent the static pressure and dynamic pressure, respectively. $\tau$ is the shear stress. The first term is the vorticity tilting or stretching term, which vanishes for the $2 \mathrm{D}$ vertical problem. If diffusion is neglected, which is true in the simulation with a small viscosity coefficient, vorticity is generated by the cross-product of the density gradient and the gradient of the static pressure (static pressure production, the second term) and dynamic pressure (dynamic pressure production, the third term), respectively. Fig. 7 shows snapshots of the ratio of the dynamic pressure production to the static pressure production at $t=6,13$ and $20 \mathrm{~s}$. The static pressure production is generally $O(10)-O\left(10^{5}\right)$ order of magnitude larger than the dynamic pressure production. The maximum value of the dynamic pressure production is at least an order of magnitude smaller than the static pressure production during the entire simulation, suggesting that the static pressure production plays a dominant role in vorticity 
generation. The accuracy in modeling the baroclinic force depends on the accuracy in predicting density distributions and thus is closely related to the mass transport advected by the current velocity field. It may be the reason why the calculation of velocity and baroclinic pressure requires a higher resolution than the dynamic pressure calculation. However, the comparison between the non-hydrostatic model and the hydrostatic model (Fig. 3(d)) indicated that the dynamic pressure production plays an important role in triggering the Kelvin-Helmholtz instability, and thus is also an important component in modeling the physical process.

\subsection{An internal solitary wave shoaling and breaking over a sloping bottom}

Mixing induced by the breaking of internal waves on sloping boundaries plays an important role in controlling the mass transport and the dissipation of internal wave energy in stratified fluids. The case of an internal wave breaking on a slope is to test whether the PDI method can preserve the model's capability for simulating the process of internal solitary wave breaking on a uniform slope. The corresponding laboratory experiment was conducted by Michallet and Ivey [1999].

The computational domain (Fig. 8) is a 2D rectangular flume with a length of $165 \mathrm{~cm}$, which is discretized by 660 grids in the horizontal direction. The water depth remains constant at $H=15 \mathrm{~cm}$ from the left boundary $(-102 \mathrm{~cm})$ to the toe of the slope and then linearly decreases from this position to the right boundary with a slope $s=0.214$. Five numerical scenarios 
with $(200,200),(200,20),(100,20),(50,20)$ and $(20,20)$ vertical layers were calculated. The initial density profile is adopted from Bourgault and Kelley [2004] and is given by

$$
\rho=\rho_{1}+\frac{\Delta \rho}{2}\left\{1+\tanh \left(\frac{z-z_{i}-\zeta}{\Delta h}\right)\right\}
$$

where $\rho_{1}=1000 \mathrm{~kg} \mathrm{~m}^{-3}$ is the density in the upper layer, $\Delta \rho=12 \mathrm{~kg} \mathrm{~m}^{-3}$, $\rho_{2}=\rho_{1}+\Delta \rho$ is the density of the lower layer, $z_{i}=0.16 H$ is the depth of the interface, $\Delta h=1.4 \mathrm{~cm}$ is the thickness of the pycnocline, $\zeta$ is the vertical displacement of the initial isopycnals and is given by

$$
\zeta=2 a_{0} \operatorname{sech}^{2}\left[\frac{\left(x-x_{0}\right)}{2 W}\right]
$$

where $a_{0}=3.1 \mathrm{~cm}, x_{0}$ is the origin which is $-102 \mathrm{~cm}$ in this case, and $W$ is the half width defined as

$$
W=\frac{2 h_{1} h_{2}}{\sqrt{3 a_{0}\left(h_{2}-h_{1}\right)}}
$$

where $h_{1}$ and $h_{2}$ are the upper and lower layer thickness, respectively, and $h_{1}=0.024 \mathrm{~m}$ and $h_{2}=0.126 \mathrm{~m}$. The model was run with a constant viscosity and diffusivity of $10^{-6} \mathrm{~m}^{2} / \mathrm{s}$. A constant bottom roughness height $(0.001 \mathrm{~m})$ was used in the bottom boundary condition.

Fig. 9 illustrates snapshots of the density distribution and velocity fields using the $(200,200)$ grid configuration. As the internal wave approaches the 
slope, the volume of the lower layer fluid reduces and the front half of the wave becomes parallel to the slope as shown in Fig. 9 (b), which increases the down-slope velocities near the bottom. In the back half of the wave, velocities cross the interface perpendicularly, causing the wave breaking and the generation of a bolus as shown in Fig. 9 (c).

Fig. 10 compares the velocity and density distributions obtained by the physical experiment and the model with the $(200,200)$ grid configuration. The model can capture the formation and evolution of the bolus, with the position of the bolus and the velocity field comparable to the measurement.

Fig. 11 shows the comparison of the velocity and density distributions between cases of $(20,20)$ and $(200,20)$ layers at $t=33.5,34.6$ and $36 \mathrm{~s}$, respectively. Compared with the full grid model shown in Fig. 10, the size and position of the bolus cannot be well resolved by the $(20,20)$ layers model, indicating that more vertical layers are needed to retain consistency with the full grid model. It can also be seen that the results of $(200,20)$ layers are much closer to those of $(200,200)$ layers. There is no apparent difference in the density and velocity distributions between the results of $(200,200)$ and $(200,20)$ layers case.

Figs. 12 and 13 show comparisons of the horizontal velocity $u$, salinity $S$, vertical velocity $w$ and dynamic pressure $p$ between the full grid model with $(200,200)$ and $(20,20)$ layers and the PDI models with $(200,20),(100,20)$ and $(50,20)$ layers at two sections at $x=33$ and $x=40 \mathrm{~cm}$, at time $t=$ 33.5 s. The results from the model with $(200,20)$ layers fit well with that 
from the full grid model with $(200,200)$ layers for all compared variables. The comparisons also show that, as the resolution of velocity/tracer grid decreases, the variables predicted by the PDI model deviate from the full grid model, similar to the trend found in the lock exchange case. Again, the deviation increases with decrease in the velocity/tracer grid resolution.

\subsection{A two-layer flow over a fixed sill}

The problem of two layer flow over topography has been studied in geophysical fluid mechanics over several decades since the work of Queney [1948]. In estuaries, stratified flows can develop in area with relative high river discharges and small tidal oscillations. The strong stratified flows can be simplified as two layer flows in the laboratory experiments and numerical modeling. Lawrence [1993] conducted a laboratory experiment on steady two-layer flow over a fixed obstacle. The experiment emphasized a flow regime of a two-layer flow, called approach-controlled flow, where an internal hydraulic control is located near the foot of the obstacle (supercritical leap, Baines, 1995). In the approach-controlled flow, the interface level around the downstream of the obstacle crest drops rapidly, with the flow changing from a supercritical flow where the upper layer is thinner to one where the lower layer is thinner. The transition involves significant non-hydrostatic processes and is not predicted by the traditional hydraulic theory (Lawrence, 1993).

The approach-controlled flow is supercritical along the sill and a hydraulic jump occurs, which can involve 3D structures to the flow at the lee side of 
the sill. A 2D model would underestimate the turbulence induced by the jump and cannot predict 3D turbulence coherence structures. Here, we use models with and without the PDI method to demonstrate the efficiency and accuracy of the PDI method in a 3D simulation.

Fig. 14 shows a schematic diagram of the experiment. The center of the sill is located at $x=0$. The bathymetry is uniform in the $y$ direction (cross-section). The two-layer flow with the density $\rho_{1}$ in the upper layer and $\rho_{2}$ in the lower layer move from left to right with the flux rates $q_{1}$ and $q_{2}$, respectively. Following the experimental setup, the length and width of the computational domain are specified as $20 \mathrm{~m}$ and $0.38 \mathrm{~m}$, respectively. The shape of the sill satisfies the following equation:

$$
h(x)=h_{m} \cos ^{2}(x / L)
$$

where $h_{m}=0.15 \mathrm{~m}$, the length scale $L=4 h_{m}$ and the range $|x / L| \leq \frac{1}{2} \pi$.

The computational grid has a uniform resolution of $\triangle x=0.05 \mathrm{~m}$ and $\triangle y=0.0475 \mathrm{~m}$ in the horizontal directions. In the vertical direction, three grid configurations are set up with $(40,40)$ for the full grid model, $(40,20)$ and $(40,10)$ for the PDI models.

Based on the experimental setup for the regime of the approach-controlled flow (Regime III, case 21 in Lawrence, 1993), the initial density profile in the 
487

model is described as

$$
\rho=\rho_{0}+\frac{\Delta \rho}{2}\left[1+\tanh \left(\frac{z-h_{1}}{\Delta h}\right)\right]
$$

488

where $\rho_{0}=1000 \mathrm{~kg} / \mathrm{m}^{3}$ is the density of freshwater in the upper layer, $\Delta \rho=$ $8.98 \mathrm{~kg} / \mathrm{m}^{3}$ is density difference between the two layers; $h_{1}=h_{2}=25.55 \mathrm{~cm}$ are the thicknesses of the upper layer and the lower layer, respectively. The density interface thickness $(\Delta h)$ is $1 \mathrm{~cm}$ for a smooth transition at the interface. The flow rates of the upper layer and the lower layer are $q_{1}=204$ $\mathrm{cm}^{2} / \mathrm{s}$ and $q_{2}=205 \mathrm{~cm}^{2} / \mathrm{s}$, respectively. The simulation is carried out using a LES approach, which is the Smagorinsky model( Smagorinsky, 1963). The total simulation time is $300 \mathrm{~s}$ and the time step $\Delta t$ is adaptive based on the CFL criterion.

Fig. 15 shows the interfacial positions along the $x$ direction predicted by the full grid non-hydrostatic model, the hydrostatic model, and the PDI models with the two grid configurations in comparison to the data. The interfacial positions are determined by finding the maximum vertical density gradient in the computational domain as the flow reached the steady state at $t=200 \mathrm{~s}$, and are averaged over the $y$ direction. All non-hydrostatic models successfully reproduced the internal hydraulic jump (supercritical leap) occurring as the flow passes through the lee side of the sill, matching the laboratory data very well. The interfaces predicted by the full grid model and the PDI models are almost identical, indicating that the PDI model with 
fewer pressure layers can successfully capture the supercritical leap. The result from the hydrostatic model derivates significantly from the data as well as the non-hydrostatic results at the jump location, indicating the important role of the dynamic pressure in the regime of the approach-controlled flow. The non-hydrostatic model revealed significant 3D turbulence distributions and coherent structures not shown here due to scope of the paper.

Fig. 16 shows the comparisons of velocity profile between the experimental data and the numerical results upstream of the obstacle crest at $x=-1 \mathrm{~m}$, and downstream at $x=1 \mathrm{~m}$ and $x=5 \mathrm{~m}$, respectively. The quantities were obtained by averaging the values along the $y$ direction at $t=200 \mathrm{~s}$. Generally good agreements with the data are achieved at all three locations for all models. Some discrepancy between the model with the $(40,10)$ grid configuration and other models is noticeable at $x=1 \mathrm{~m}$ but is small. At $x=5 \mathrm{~m}$, the models agree well with each other but some under-prediction can be found in the lower layer in all models compared with the data. The reason for the under-prediction is unknown.

Fig. 17 shows the $y$-averaged density profiles at the same locations and times as the velocity profiles in comparison to the measurements. All models reproduced the transition from the supercritical flow with a thinner upper layer to subcritical flow with the thicker upper layer as the two-layer flow passes over the sill, with good agreement with the data. The results of hydrostatic simulations are also shown in Figs. 16 and 17. Compared with the results of non-hydrostatic model, hydrostatic model cannot predict the 
evolution of the hydraulic jump well, which is consistent with the comparison in Fig. 15.

Overall, the 3D models with the PDI method are able to capture the internal hydraulic jump and flow features in the approach-controlled flow regime with the similar accuracy with the full grid model. The tests also indicated that the predictions from the PDI models deviate from the full grid model as the resolution of the pressure grid decreases. However, decreasing the pressure layers from 40 to 10 does not affect the model predictions of velocity and density fields. Detailed model results with more extensive analysis and physical explanations will be reported later in a new paper.

\section{Discussion}

\subsection{Interpolation method}

van Reeuwijk [2002] carried out an extensive investigation of the interpolation method used in his version of a PDI method. The interpolation method was constrained to fulfill four conditions: 1) zero dynamic pressure at free surface, 2) zero dynamic pressure gradient at the bottom, 3) consistency condition and 4) piecewise monotonic pressure distribution in each pressure layer. It was found that first- and fourth-order spline interpolations satisfy the four conditions and that the fourth-order spline interpolation provided more accurate results in surface wave tests. In the present study, we focus on applications to stratified flows and implemented cubic spline and fourth-order spline interpolation methods, respectively, where the fourth- 
order follows van Reeuwijk. The cubic spline interpolation used here matches the values at both ends of the coarse grid points, ensuring zero-pressure at the surface. The cubic spline interpolation does not satisfy the piecewise monotonic condition. However, our numerical experiments in the test cases indicated the cubic spline interpolation used in PDI provided better fits to the original full grid model compared to the fourth-order spline interpolation (not shown in the paper). The fourth-order spline interpolation encountered a stability problem as the velocity-pressure grid ratio became large. The reason causing the numerical stability problem is unknown.

\subsection{Accuracy}

The idea of PDI is based on the fact that an accurate prediction of wave dispersion associated with the dynamic pressure field does not require a large number of vertical layers. Our hypothesis is that the velocity field corrected by the dynamic pressure field is weakly dependent on the vertical resolution of the dynamic pressure. Therefore, it is necessary to check the accuracy of PDI in predicting dynamic pressure and its influence on predicting velocity field and distribution of tracer variables. Here, we set the case with the $(200,200)$ grid configuration as the baseline case for the lock exchange problem and the application of internal wave breaking. The baseline of the internal hydraulic jump case is the scenario with $(40,40)$ layers. The model accuracy in predicting dynamic pressure, velocity $(u, w)$ and salinity is evaluated using the normalized root-mean-square error (NRMSE) with respect 
574

to the results of the baseline case. The NRMSE formula is defined as

$$
\operatorname{NRMSE}(t)=\sqrt{\frac{\sum_{i=1}^{N}\left(X_{i}-X_{i}^{\prime}\right)^{2}}{\sum_{i=1}^{N} X_{i}^{\prime 2}}}
$$

575

576

where $N$ is the number of data, $X_{i}^{\prime}$ represents the vertical mean result at point $i$ in the baseline case, $X_{i}$ is the vertical mean value from the cases with different grid configurations. The data are collected from all points of the grid configurations at $t=20 \mathrm{~s}$ for the lock exchange problem, $t=30 \mathrm{~s}$ for the internal wave breaking case and $t=100 \mathrm{~s}$ for the internal hydraulic jump case. Table 1,2 and 3 list the NRMSE of the three cases. All cases show that NRMSE decreases with increase of the number of vertical pressure layers. More-over, NRMSE also decreases with the increase of vertical velocity layers (tests for different velocity layers are not conducted in the internal hydraulic jump case).

Different interpolation methods in PDI, including the linear, quadratic and cubic spine interpolation, were examined in all test cases. Results show that the cubic spline interpolation gives the most accurate estimation in terms of NRMSE.

\subsection{Efficiency}

In order to eliminate the differences caused by different parallel computing configurations, we calculated all cases using a single processor. The improvement in model efficiency was evaluated by comparing the computational time used in solving the Poisson equation in the full grid model versus 
the time used in solving the Poisson equation plus the overhead associated with the interpolations in the PDI model. Fig. 18 shows the computational ratio $\left(r_{p}\right)$ which is defined as

$$
r_{p}=\frac{t}{t_{20}}
$$

where $t_{20}$ is the computational time for the $(20,20)$ layers case.

Notice that the computational time in the cases without PDI increases linearly with the increase of velocity and pressure layer numbers. Increasing the number of vertical layers from $(20,20)$ to $(200,200)$ results in 9.4 and 8.5 times rise in computational time for the lock exchange problem and internal wave breaking case, respectively. However, the computational ratio in the cases with PDI method remains within 1.5 for the lock exchange case and 1.3 for the internal wave case when the vertical grid configuration changes from $(20,20)$ to $(200,20)$. The increased computational time is associated with the interpolation, thus the overhead of the PDI method is two times less than that of solving Poisson equation in the cases with vertical grid configuration of $(200,20)$.

Tests on the 3D case of the internal hydraulic jump also show that the PDI method can greatly improve the model's efficiency. The computational time in solving the Poisson equation reduces by $80 \%$ and $56 \%$ in the cases with the pressure layer number decreased by $75 \%$ and $50 \%$, respectively. Because the 3D case uses different grid configurations from the 2D cases, the 
computational time ratio $r_{p}$ is not included in Fig. 18.

\subsection{Convergence}

Numerical convergence rates usually depend on the order of accuracy of a numerical scheme and can be examined by grid refinement and time refinement. The model convergence with respect to the pressure layer refinement in the PDI method is different from general numerical convergence (usually evaluated by convergence rates with respect to mesh refinement and time step refinement) because it not only depends on the numerical scheme but also is related to physics modeled in a specific case. A model convergence test for the PDI method can examine what degree of pressure decimation can affect non-hydrostatic processes. The NRMSE in Table 1-3 decreases with increase of the vertical pressure layer number, which has indicated that the PDI method is convergent to the full grid model. To further test the model convergence with respect to the pressure layer refinement, we carried out extra tests with different $n_{p}$ in the test cases. We did not calculate the convergence rate which is usually obtained by integrating computational errors over the whole computational domain and used to evaluate orders of numerical accuracy. Instead, we use a single point-comparison to demonstrate the model convergence with the pressure layer refinement. Fig. 19 shows the vertical pressure distributions at $x=0.4 \mathrm{~m}$ and $t=5 \mathrm{~s}$ from the PDI model with $n_{p}=10,15$, and 20 , in comparison to the full grid model $\left(n_{p}=200\right)$ for the case of the lock exchange problem. The figure shows that the vertical 
distribution of dynamic pressure from the PDI models are approaching that of the full grid model with increasing $n_{p}$, indicating the convergence of the PDI model. It is also noted that the model results from $n_{p}=15$ and 10 deviate largely from the PDI model with $n_{p}=20$ and the full grid model even at the beginning of the simulation $(t=5 \mathrm{~s})$, and the differences become more significant for later time. Our tests with $n_{p}=50$ and 100 provide similar results as the test with $n_{p}=20$ (Table 1 and 2), suggesting that the PDI model converges at $n_{p}=20$ for the case. Fig. 20 shows the same comparisons for the case of the internal wave breaking at $x=-65 \mathrm{~cm}$ and $t$ $=5 \mathrm{~s}$. The same conclusion can be drawn. The model convergence for the case of the internal hydraulic jump is demonstrated in the comparisons of the dynamic pressure distributions at $x=1 \mathrm{~m}$ and at the steady state as shown in Fig. 21. Different from the other two cases, the differences in the pressure distribution between the PDI models and the full grid model are generally small, but the tendency of convergence can still be found as $n_{p}$ increasing from 10 to 40 .

As indicated in the three test cases, the model convergence with respect to the pressure layer refinement is highly related to specific physical processes. The convergence rates can change case by case and depend on a specific nonhydrostatic process. We carried out the pressure decimation on the basis of trial-and-error. The question how to select the number of the pressure layers in the PDI model is not answered in the present study. Further work is needed to explain why the dynamic pressure can be decimated in a non-hydrostatic 
model for simulating baroclinic, non-hydrostatic flows.

\subsection{PDI in horizontal direction}

The extension of the PDI method in the horizontal direction was examined using the lock exchange case as an example. The test was done in the similar procedures as the PDI in the vertical direction but reducing the horizontal resolution of the pressure grid in contrast to the vertical resolution in the vertical PDI.

The case was run by reducing $50 \%$ of the horizontal resolution of pressure grid and keeping the same number of vertical pressure layers as the full grid model (200 layers) in order to examine the influence from the horizontal PDI method only. The flow patterns predicted by the model with the horizontal PDI are similar to the full grid model (not shown here). However, further reducing the horizontal pressure grid resolution did not yield flow patterns comparable to the full grid model. The test may indicate that the effects of dynamic pressure gradient are more significant in the horizontal direction than in the vertical direction in the non-hydrostatic, baroclinic processes.

\section{Conclusions}

A pressure decimation and interpolation method was applied in the nonhydrostatic wave model NHWAVE in order to improve the computational efficiency. The idea was to use a coarser grid in solving the pressure Poisson equation and a finer grid in the rest of equations for velocity and passive 
tracers. With a lower resolution grid in solving the Poisson equation, the computational efficiency was greatly improved.

Test cases in the study include simulations of the lock exchange problem, the internal waves breaking and a two-layer flow over a fixed sill, all of which used several grid configurations. The cases showed that a considerable large number of vertical layers is necessary for resolving the velocity structure and density field for modeling non-hydrostatic, stratified flows. The results indicated that the reduction of vertical layer number in solving the Poisson equation did not obviously alter the numerical results but improved computational efficiency significantly. The applications of the horizontal PDI to the test cases did not show much improvement in model efficiency compared to PDI in the vertical direction.

Acknowledgements The authors wish to thank Dr. Stephan Kramer and two anonymous reviewers for their constructive comments and suggestions. This work was supported by the National Science Foundation, Physical Oceanography Program (OCE-1334325 and OCE-1435147) and the Office of Naval Research, Littoral Geosciences and Optics Program (N00014-10-10188) and Arctic and Global Prediction Program(N00014-14-1-0569) (Kirby and F. Shi). J. Shi and G. Wu were supported by the National Natural Science Foundation of China (51339005), the National Science Foundation for Distinguished Young Scholars (51425901) and 111 Project through Grant No. B14028. Development and testing of the parallel code was carried out 
on UD's Community Cluster System operated by UD IT group, supported by NSF award CNS-0958512.

\section{References}

Auclair, F., Estournel, C., Floor, J.W., Herrmann, M., Nguyen, C. and Marsaleix, P., 2011. A non-hydrostatic algorithm for free-surface ocean modelling, Ocean Modelling, 36, 49-70, doi: 10.1016/j.ocemod.2010.09.006.

Baines, P.G.,1995. Topographic Effects in Stratified Flows, Cambridge Univ. Press, 482 pp.

Berntsen, J., Furnes, G., 2005. Internal pressure errors in sigma-coordinate ocean models-sensitivity of the growth of the flow to the time stepping method and possible non-hydrostatic effects, Continental Shelf Research, 25, 829-848, doi: 10.1016/j.csr.2004.09.025.

Bourgault, D., and Kelley, D.E., 2004. A laterally averaged nonhydrostatic ocean model, J. Atmos. Oceanic Technol., 21 (12), 1910-1924, doi:10.1175/JTECH-1674.1.

Bradford, S., 2011. Nonhydrostatic model for surf zone simulation, J. Waterway, Port, Coastal, Ocean Eng, 137, 163-174, doi: 10.1061/(ASCE) WW.1943-5460.0000079.

Burchard, H. and Bolding. K., 2002. GETM C A general estuarine transport model. Scientific Documentation. Technical Report EUR 20253 
Casulli, V., and Stelling, G., 1998. Numerical smulation of 3D quasihydrostatic, free-surface flows, J. Hydraul. Eng., 124, 678-686, doi:10.10 61(ASCE)0733-9429(1998)124:7(678)

Casulli, V., 1999. A semi-implicit finite difference method for non-hydrostatic, free-surface flows, Int. J. Numer. Meth. Fluids, 30(4), 425-440, doi: 10.1002/(SICI)1097-0363(19990630)30:4<425::AID-FLD847>3.0.CO;2D.

Cui, H., 2013, A new numerical model for simulating the propagation of and inundation by tsunami waves, Ph.D. Thesis, Delft University of Technology, Netherland, doi:10.4233/uuid:1440c86c-2e53-4a3c-a43c77 ef9b75fa82.

Cui, H., Pietrzak, J. and Stelling, G., 2014, Optimal dispersion with minimized Poisson equations for non-hydrostatic free surface flows, Ocean Modelling, 81, 1-12, doi:10.1016/j.ocemod.2014.06.004.

Chen, C., Liu, H. and Beardsley, R.C., 2003. An unstructured grid, finitevolume, three-dimensional, primitive equations ocean model: Application to coastal ocean and estuaries, J. Atmos. Oceanic Technol., 20 (1), 159-186, doi:10.1175/1520-0426(2003)020<0159:AUGFVT>2.0.CO;2.

Chen, X., 2003. A fully hydrodynamic model for three-dimensional, freesurface flows, Int. J. Numer. Meth. Fluids, 42 (9), 929-952. 
Dietrich D. and Lin, C.A., 2002. Effects of hydrostatic approximation and resolution on the simulation of convective adjustment, Tellus A, Munksgaard International Publishers, 54, 34-43, doi: 10.1034/j.16000870.2002.00162.x.

Fringer, O., Gerritsen, M. and Street, R., 2006. An unstructured-grid, finite-volume, nonhydrostatic, parallel coastal ocean simulator, Ocean Modelling, 14, 139-173, doi: 10.1016/j.ocemod.2006.03.006.

Gaarthuis, J., 1995. A non-hydrostatic pressure model for shallow water flow: Analysis and application of three methods to compute a nonhydrostatic correction term, Ph.D. Thesis, Technische University, Einshoven.

Goda, K., 1979. A multistep technique with implicit difference schemes for calculating two- or three-dimensional cavity flows, J. Comput. Phys, 30, 76-95, doi: 10.1016/0021-9991(79)90088-3.

Gottlieb, S., Shu, C.W. and Tadmor, E., 2001. Strong stability-preserving high-order time discretization methods, SIAM Rev, 43, 89-112.

Guermond, J., Minev, P. and Shen, J., 2006. An overview of projection methods for incompressible flows, Computer Methods in Applied Mechanics and Engineering, 195, 6011-6045, doi: 10.1016/j.cma.2005.10.010.

Harlow, F.H. and Welch, J.E., 1965. Numerical calculation of time-dependent viscous incompressible flow of fluid with free surface, Physics of Fluids, 
8, 2182-2189, doi: 10.1063/1.1761178.

Heggelund, Y., Vikebø, F., Berntsen, J. and Furnes, G., 2004. Hydrostatic and non-hydrostatic studies of gravitational adjustment over a slope, Continental Shelf Research, 24, 2133-2148, doi: 10.1016/j.csr.2004.07.005.

Hirt, C. and Nichols, B., 1981. Volume of fluid (VOF) method for the dynamics of free boundaries, Journal of Computational Physics, 39, 201-225, doi: 10.1016/0021-9991(81)90145-5.

Jankowski J.A., 1999. A non-hydrostatic model for free surface flows. Ph.D. Thesis, Hannover University, Germany.

Johns, B., 1991. The modelling of the free surface flow of water over topography, Coastal Engineering, 15, 257-278, doi: 10.1016/0378-3839(91)900 $05-2$.

Johns, B. and Xing , J., 1993. Three-dimensional modelling of the free surface turbulent flow of water over a bedform, Continental Shelf Research, 13, 705-721, doi: 10.1016/0278-4343(93)90024-R.

Kanarska, Y. and Maderich, V., 2003. A non-hydrostatic numerical model for calculating free-surface stratified flows, Ocean Dynamics, 53, 176185, doi: 10.1007/s10236-003-0039-6.

Kanarska, Y., Shchepetkin, A. and McWilliams, J., 2007. Algorithm for non-hydrostatic dynamics in the Regional Oceanic Modeling System, Ocean Modelling, 18, 143-174, doi: 10.1016/j.ocemod.2007.04.001. 
Keilegavlen, E. and Berntsen, J., 2009. Non-hydrostatic pressure in $\sigma$ coordinate ocean models, Ocean Modelling, 28, 240-249, doi:10.1016/j. ocemod.2009.02.006.

Kim, D.H., and Lynett, P.J.,2011. Turbulent mixing and passive scalar transport in shallow flows, Physics of Fluids, 23, 016603, doi: 10.1063/1.3 531716.

Kim, D.H., Lynett, P.J., and Socolofsky, S.A., 2009. A depth-integrated model for weakly dispersive, turbulent, and rotational fluid flows, Ocean Model, 27, 198-214, doi: 10.1016/j.ocemod.2009.01.005.

Klingbeil, K. and Burchard, H., 2013. Implementation of a direct nonhydrostatic pressure gradient discretisation into a layered ocean model, Ocean Modelling, 65, 64-77, doi: 10.1016/j.ocemod.2013.02.002.

Lai, Z., Chen, C., Cowles, G.W. and Beardsley, R.C., 2010a. A nonhydrostatic version of FVCOM: 1. Validation experiments, J. Geophys. Res., 115, C11010, doi: 10.1029/2009JC005525.

Lai, Z., Chen, C., Cowles, G.W. and Beardsley, R.C., 2010b. A nonhydrostatic version of FVCOM: 2. Mechanistic study of tidally generated nonlinear internal waves in Massachusetts Bay, J. Geophys. Res., 115, C12049, doi:10.1029/2010JC006331.

Lawrence, G. A., 1993. The hydraulics of steady two-layer flow over a fixed obstacle. J. Fluid Mech, 254, pp 605-633. 
Li, Z. and Johns, B., 2001. A numerical method for the determination of weakly non-hydrostatic non-linear free surface wave propagation, Int. J. Numer. Meth. Fluids, 35, 299-317, doi: 10.1002/1097-0363(20010215) 35:3<299::AID-FLD94>3.0.CO;2-B.

Ma, G., Shi, F. and Kirby, J.T., 2012. Shock-capturing non-hydrostatic model for fully dispersive surface wave processes, Ocean Modelling, 4344 , 22 - 35, doi: 10.1016/j.ocemod.2011.12.002.

Ma, G., Kirby, J.T. and Shi, F., 2013. Numerical simulation of tsunami waves generated by deformable submarine landslides, Ocean Modelling, 69, 146 - 165, doi: 10.1016/j.ocemod.2013.07.001.

Mahadevan, A., Oliger, J. and Street, R., 1996. A nonhydrostatic mesoscale ocean model. Part i: Well-posedness and scaling, J. Phys. Oceanogr., 26 (9), 1868-1880, doi:10.1175/1520-0485(1996)026<1868:ANMOMP> 2.0. CO;2.

Michallet, H. and Ivey, G.N., 1999. Experiments on mixing due to internal solitary waves breaking on uniform slopes, J. Geophys. Res., 104 (C6), 13,467-13,477, doi: 10.1029/1999JC900037.

Namin, M.M., Lin, B., and Falconer, R.A., 2001. An implicit numerical algorithm for solving non-hydrostatic free-surface flow problems, Int. J. Numer. Meth. Fluids, 35 (3), 341-356, doi: 10.1002/10970363(20010215)35:3<341::AID-FLD96>3.0.CO;2-R. 
Pope, S., 2000. Turbulent Flows, Cambridge University Press, 522pp

Queney, P., 1948. The problem of air flow over mountains: A summary of theoretical results, Bull. AMS, 29, 16-26.

Roe, P.L.,1986. Characteristic-based schemes for the Euler equations, Ann. Rev. Fluid Mech. 18, 337-365.

Scotti, A. and Mitran, S.,2008. An approximated method for the solution of elliptic problems in thin domains: Application to nonlinear internal waves. Ocean Modelling, 25, 144-153, doi:10.1016/j.ocemod.2008.07.005.

Shchepetkin, A.F., and McWilliams, J.C., 2005. The regional oceanic modeling system (ROMS): a split-explicit, free-surface, topographyfollowing-coordinate oceanic model, Ocean Modelling, 9 (4), 347-404, doi:10.1016/j.ocemod.2004.08.002.

Shi, F., Kirby, J.T., Harris, J.C., Geiman, J.D., and Grilli, S.T., 2012. A high-order adaptive timestepping TVD solver for Boussinesq modeling of breaking waves and coastal inundation, Ocean Modelling, 43-44, 3651, doi: 10.1016/j.ocemod.2011.12.004.

Smagorinsky, J., 1963. General circulation experiments with the primitive equations: I. the basic experiment*, Monthly weather review, 91(3), 99-164.

Stelling, G.W., 1984. On the construction of computational methods for 
shallow water flow problems, Technical Report 35, Rijkwaterstaat, Netherlands.

Stelling, G.W., 1986. Practical aspects of accurate tidal computations, J. Hydr. Eng, 112, 802-817, doi: 10.1061/(ASCE)0733-9429(1986)112:9(802).

Stelling, G. and Zijlema, M., 2003. An accurate and efficient finite-difference algorithm for non-hydrostatic free-surface flow with application to wave propagation, Int. J. Numer. Meth. Fluids,43, 1-23, doi: 10.1002/fld.595

van Kan, J., 1986. A second-order accurate pressure correction scheme for viscous incompressible flow,SIAM J. Sci. Stat. Comput., 7, 870-891, doi:10.1137/0907059.

van Leer, B., 1974. Towards the ultimate conservative difference scheme II. Monotonicity and conservation combined in a second order scheme, $J$. Comp. Phys. 14 (4): 361-370.

van Reeuwijk, M. 2002. Efficient simulation of non-hydrostatic free-surface flow, Master's thesis, Delft University of Technology, Netherlands.

Vitousek, S. and Fringer, O. B., 2014. A nonhydrostatic, isopycnal-coordinate ocean model for internal waves, Ocean Modelling, 83, 118-144, doi:10.1016 /j.ocemod.2014.08.008.

Young, C.-C. and Wu, C.H., 2009. An efficient and accurate non-hydrostatic model with embedded Boussinesq-type like equations for surface wave 
modeling, Int. J. Numer. Meth. Fluids, 60, 27-53, doi: 10.1002/fld.1876.

Young, C.-C. and Wu, C.H., 2010. A $\sigma$-coordinate non-hydrostatic model with embedded Boussinesq-type-like equations for modeling deep-water waves, Int. J. Numer. Meth. Fluids, 63, 1448-1470, doi: 10.1002/fld.2150.

Yuan, H. and Wu, C.H., 2004. An implicit three-dimensional fully nonhydrostatic model for free-surface flows, Int. J. Numer. Meth. Fluids, 46, 709-733, doi: 10.1002/fld.778.

Zheng, J., Yan, Y. and Zhu, Y.,2002. Three dimensional baroclinic numerical model for simulating fresh and salt water mixing in the Yangtze Estuary, China ocean Engineering, 16(2), 227-238, doi:10.3321/j.issn:08905487.2002 .02 .008 .

Zijlema, M., Stelling, G. and Smit, P., 2011. SWASH: An operational public domain code for simulating wave fields and rapidly varied flows in coastal waters, Coastal Engineering, 58, 992-1012, doi: 10.1016/j.coastal eng.2011.05.015. 
Table 1: The results of NRMSE for the lock-exchange problem

\begin{tabular}{lcccc}
\hline Case & Dynamic pressure & Salinity & $u$ & $w$ \\
\hline$(20,20)$ & $5.14 \times 10^{-2}$ & $3.73 \times 10^{-2}$ & $1.27 \times 10^{-3}$ & $4.29 \times 10^{-3}$ \\
$(50,20)$ & $3.39 \times 10^{-2}$ & $1.32 \times 10^{-2}$ & $9.73 \times 10^{-5}$ & $6.03 \times 10^{-4}$ \\
$(100,20)$ & $2.72 \times 10^{-2}$ & $7.71 \times 10^{-3}$ & $6.30 \times 10^{-5}$ & $2.71 \times 10^{-4}$ \\
$(200,20)$ & $9.41 \times 10^{-3}$ & $3.79 \times 10^{-3}$ & $2.43 \times 10^{-6}$ & $6.70 \times 10^{-5}$ \\
$(200,50)$ & $2.32 \times 10^{-3}$ & $2.51 \times 10^{-4}$ & $6.76 \times 10^{-7}$ & $5.50 \times 10^{-6}$ \\
$(200,100)$ & $3.06 \times 10^{-4}$ & $7.13 \times 10^{-5}$ & $3.61 \times 10^{-7}$ & $2.01 \times 10^{-6}$ \\
\hline
\end{tabular}

Table 2: The results of NRMSE for the internal wave breaking case

\begin{tabular}{lcccc}
\hline Case & Dynamic pressure & Salinity & $u$ & $w$ \\
\hline$(20,20)$ & $2.06 \times 10^{-1}$ & $1.80 \times 10^{-1}$ & $1.72 \times 10^{-3}$ & $2.76 \times 10^{-3}$ \\
$(50,20)$ & $9.71 \times 10^{-3}$ & $2.77 \times 10^{-3}$ & $2.31 \times 10^{-5}$ & $1.91 \times 10^{-4}$ \\
$(100,20)$ & $7.02 \times 10^{-3}$ & $1.53 \times 10^{-3}$ & $1.42 \times 10^{-5}$ & $6.01 \times 10^{-5}$ \\
$(200,20)$ & $5.46 \times 10^{-3}$ & $6.90 \times 10^{-4}$ & $8.05 \times 10^{-6}$ & $4.33 \times 10^{-5}$ \\
$(200,50)$ & $2.34 \times 10^{-3}$ & $2.70 \times 10^{-4}$ & $4.30 \times 10^{-6}$ & $8.11 \times 10^{-6}$ \\
$(200,100)$ & $6.77 \times 10^{-4}$ & $8.98 \times 10^{-5}$ & $1.01 \times 10^{-6}$ & $3.04 \times 10^{-6}$ \\
\hline
\end{tabular}

Table 3: The results of NRMSE for the internal hydraulic jump case

\begin{tabular}{lcccc}
\hline Case & Dynamic pressure & Salinity & $u$ & $w$ \\
\hline$(40,10)$ & $4.6 \times 10^{-2}$ & $1.3 \times 10^{-4}$ & $2.9 \times 10^{-4}$ & $4.4 \times 10^{-3}$ \\
$(40,20)$ & $2.4 \times 10^{-2}$ & $3.0 \times 10^{-5}$ & $2.8 \times 10^{-5}$ & $2.4 \times 10^{-3}$ \\
\hline
\end{tabular}




\section{List of Figures}

1 Grid arrangement in PDI. $p^{\prime}$ and $\left(u^{\prime}, w^{\prime}\right)$ denote the dynamic pressure and velocity points, respectively in the coarse grid. $p$ and $(u, w)$ are the dynamic pressure and velocity points in the fine grid. . . . . . . . . . . . . . . . . . . 52

2 Flow chart of PDI. . . . . . . . . . . . . . 53

3 Comparisons of density distributions for the lock exchange problem at 20.0 s. (a) $(20,20)$ layers; (b) $(200,20)$ layers; (c) $(200,200)$ layers; (d) hydrostatic simulation . . . . . . . . . . 54

4 Time series of the surface (upper panel)and bottom (lower panel) horizontal velocity at $x=0.4 \mathrm{~m}$. Dashed lines: theoretical values $(1.59 \mathrm{~cm} / \mathrm{s})$; Blue lines: (20,20) layers case; Black lines: $(200,200)$ layers case; Red lines: $(200,20)$ layers case. ........................ 54

5 Comparisons of $u$ and salinity distributions at $t=20 \mathrm{~s}$ (Blue lines: (20,20) layers; Black lines: (200,200) layers; Red-solid lines: $(200,20)$ layers; Dash-dotted lines $(100,20)$ layers; Dashed

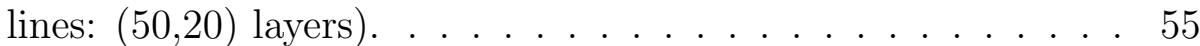

6 Comparisons of $w$ and dynamic pressure distributions at $t=$ 20 s (Blue lines: (20,20) layers; Black lines: (200,200) layers; Red-solid lines: (200,20) layers; Dash-dotted lines $(100,20)$ layers; Dashed lines: $(50,20)$ layers $) \ldots$. . . . . . . . . 56 
7 Snapshots of the ratio of the dynamic pressure production to the static pressure production at $t=6,13$ and $20 \mathrm{~s}$. . . . . 57

8 Initial density distribution of the internal wave breaking case. 58

$9 \quad$ Snapshots of simulated density and velocity fields with $(200,200)$ layers. . . . . . . . . . . . . . . . . . . . 59

10 Comparisons of velocity field and density distributions between the laboratory observation(left, Michallet and Ivey 1999) and the $(200,200)$ layers case (right). . . . . . . . . . . 60

11 Velocity and density distributions for cases of $(20,20)$ layers $\left(A_{1}, A_{2}, A_{3}\right) ;(200,20)$ layers $\left(B_{1}, B_{2}, B_{3}\right)$. . . . . . . . . 61

12 Comparisons of $u$ and salinity distributions at $t=33.5 \mathrm{~s}$ (Blue lines: (20,20) layers; Black lines: (200,200) layers; Red-solid lines: $(200,20)$ layers; Dash-dotted lines $(100,20)$ layers; Dashed lines: $(50,20)$ layers. . . . . . . . . . . . . . . . . 62

13 Comparisons of $w$ and dynamic pressure distributions at $t=$ 33.5 s(Blue lines: $(20,20)$ layers; Black lines: $(200,200)$ layers; Red-solid lines: $(200,20)$ layers; Dash-dotted lines $(100,20)$ layers; Dashed lines: $(50,20)$ layers. . . . . . . . . . . . . . 63

14 A schematic for the initial conditions in the case of the internal hydraulic jump. . . . . . . . . . . . . . . 63 
15 Comparisons of the interface positions (Black line: $(40,40)$ layers; Red line: $(40,20)$ layers; Blue line: $(40,10)$ layers; Dashed line: hydrostatic prediction; Circles: laboratory data conducted by Lawrence [1993], $Y=h(0)+\eta(x)$ is the free surface position, $h(0)$ is the depth at the west boundary) . . . . . . . . 64

16 Comparisons of the $u$ profiles at $x=-1.0 \mathrm{~m}, 1.0 \mathrm{~m}$, and $5.0 \mathrm{~m}$ (from left to right) Black lines: $(40,40)$ layers; Red lines: $(40,20)$ layers; Blue lines: $(40,10)$ layers; Dashed lines: Hydrostatic predictions; Circles: laboratory data conducted by

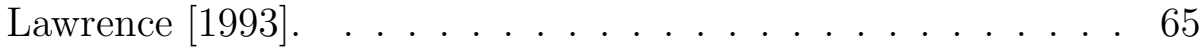

17 Comparisons of the density profile at $x=-1.0 \mathrm{~m}, 1.0 \mathrm{~m}$, and 5.0m (from left to right) Black lines: $(40,40)$ layers; Red lines: $(40,20)$ layers; Blue lines: $(40,10)$ layers; Dashed lines: Hydrostatic predictions; Circles: laboratory data conducted by

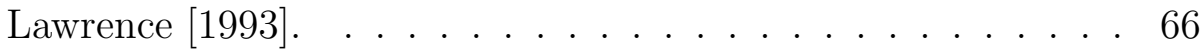

18 Computational time ratio for solving Poisson equation and interpolation. $t_{20}$ is the computational time for solving Poisson equation for the $(20,20)$ layers case. Black lines: the lock exchange case; Red lines: the internal wave case; Solid lines: the cases using the same numbers of pressure layers and velocity layers; Dashed lines: the cases with the number of $n_{p}=20 . .67$ 
19 Comparisons of dynamic pressure distributions of the lock exchange case at $t=5$ s. Black line: $(200,200)$ layers; Red line: $(200,20)$ layers; Blue line: $(200,15)$ layers; Green line: $(200,10)$ layers . . . . . . . . . . . . . . . . . . . . 68

20 Comparisons of dynamic pressure distributions of the internal wave case at $t=5 \mathrm{~s}$. Black line: $(200,200)$ layers; Red line: $(200,20)$ layers; Blue line: $(200,15)$ layers; Green line: $(200,10)$

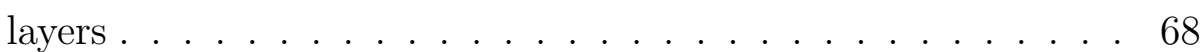

21 Comparisons of dynamic pressure distributions of the third case at $x=1.0 \mathrm{~m}$. Black line: $(40,40)$ layers; Red line: $(40,20)$ layers; Blue line: $(40,10)$ layers _. . . . . . . . . . . . . . . 69 


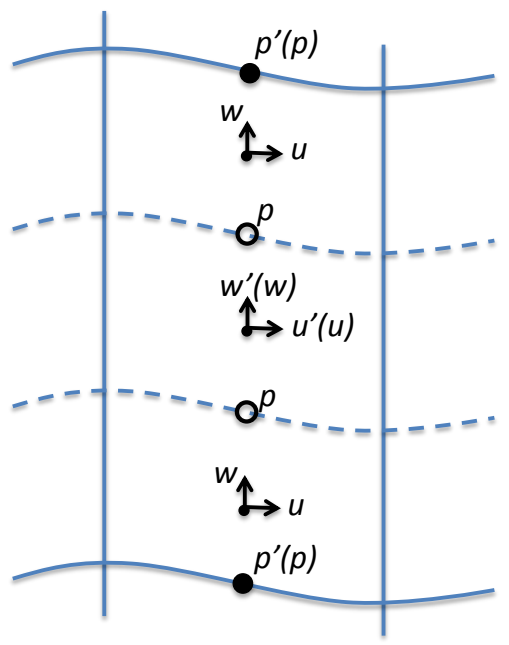

Figure 1: Grid arrangement in PDI. $p^{\prime}$ and $\left(u^{\prime}, w^{\prime}\right)$ denote the dynamic pressure and velocity points, respectively in the coarse grid. $p$ and $(u, w)$ are the dynamic pressure and velocity points in the fine grid. 


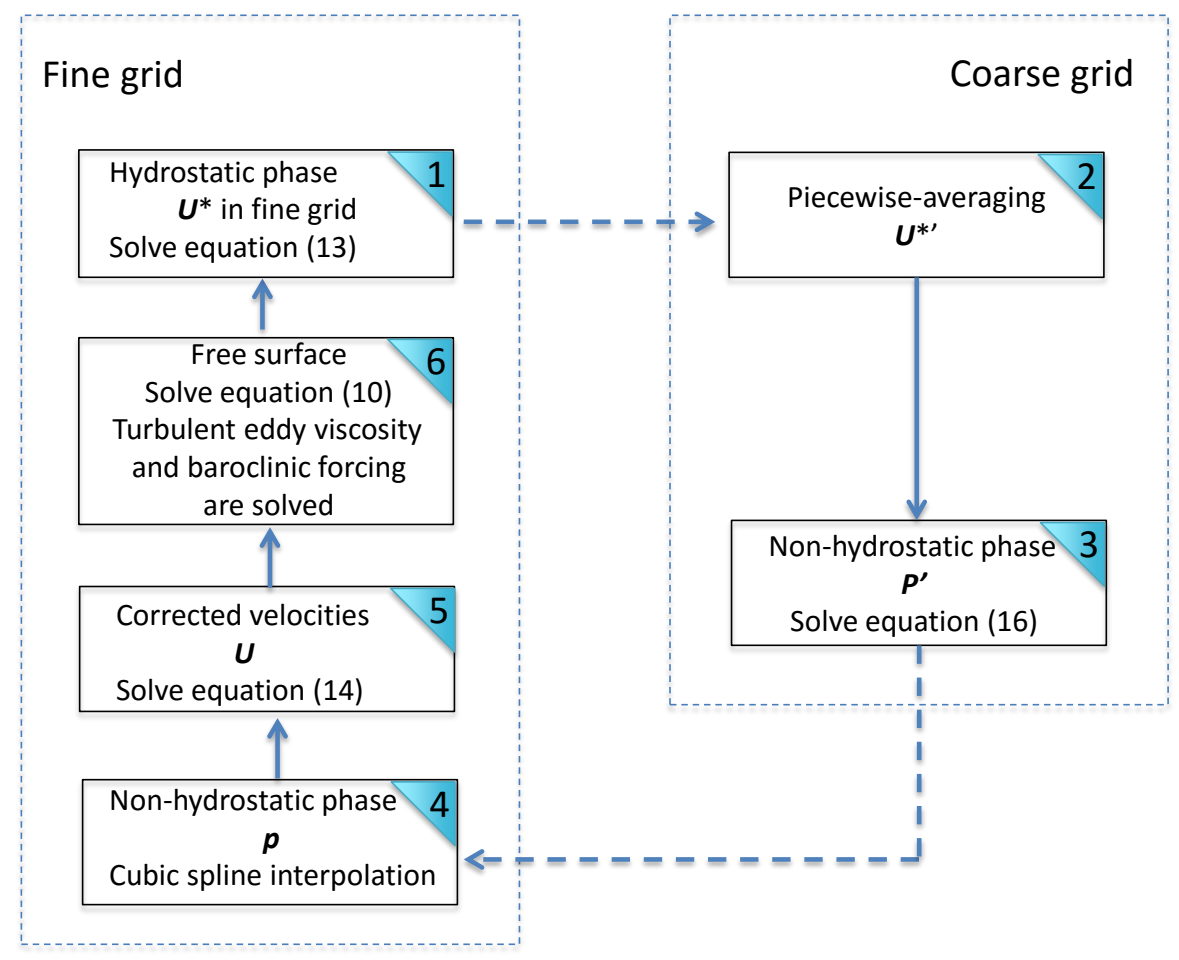

Figure 2: Flow chart of PDI. 

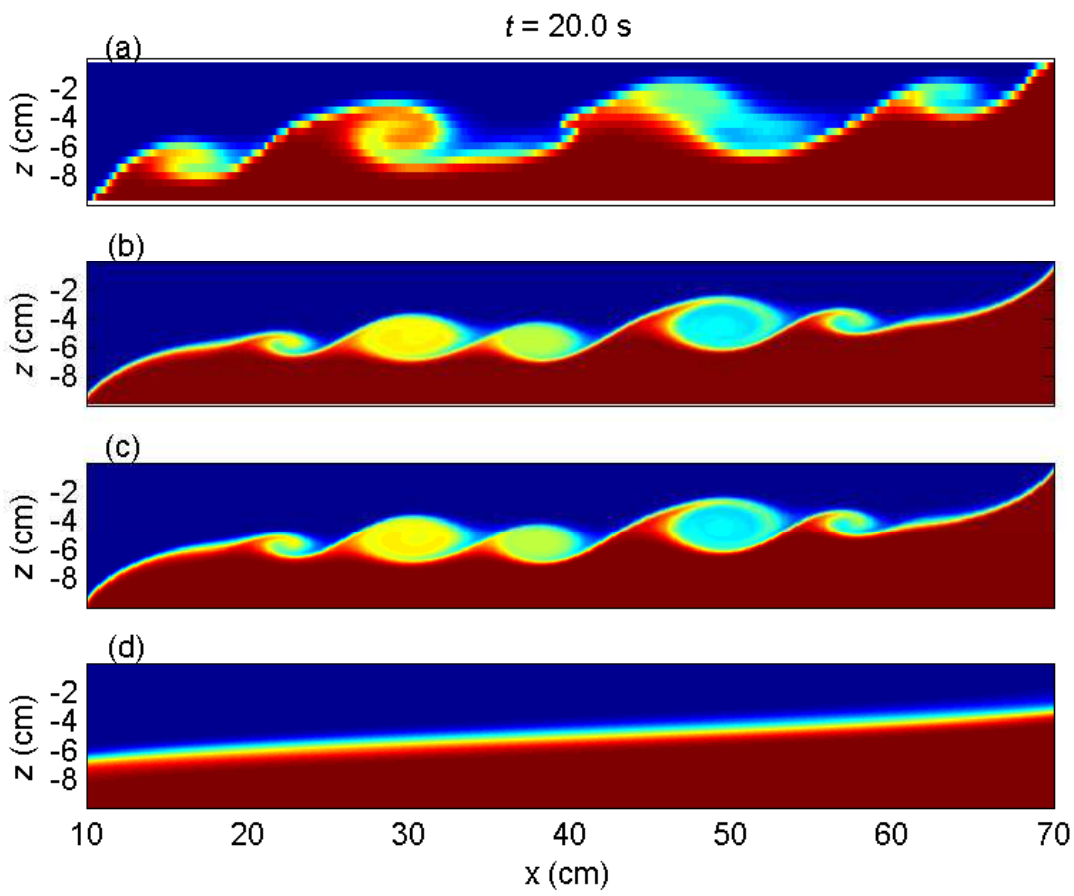

Figure 3: Comparisons of density distributions for the lock exchange problem at $20.0 \mathrm{~s}$. (a) $(20,20)$ layers; (b) $(200,20)$ layers; (c) $(200,200)$ layers; (d) hydrostatic simulation
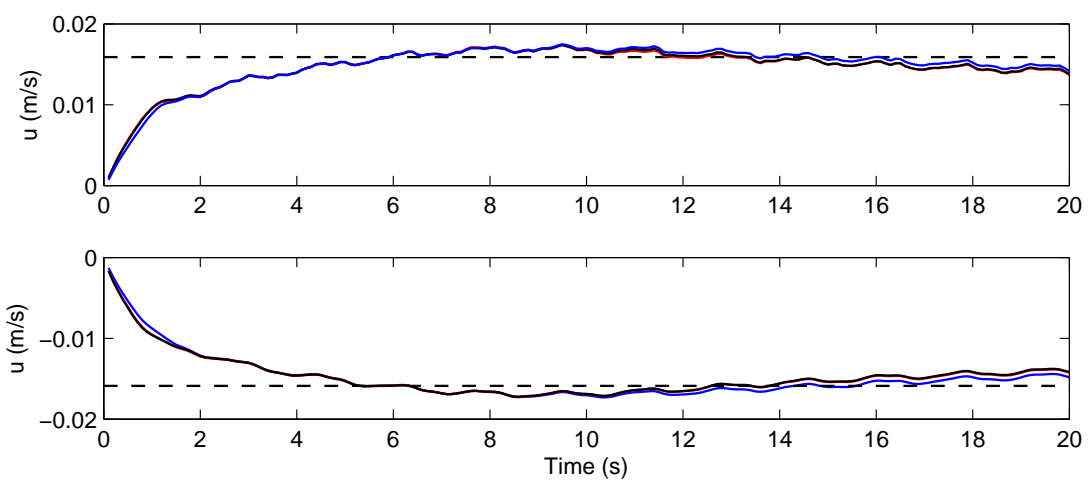

Figure 4: Time series of the surface (upper panel)and bottom (lower panel) horizontal velocity at $x=0.4 \mathrm{~m}$. Dashed lines: theoretical values $(1.59 \mathrm{~cm} / \mathrm{s})$; Blue lines: $(20,20)$ layers case; Black lines: $(200,200)$ layers case; Red lines: $(200,20)$ layers case. 
(a) $x=0.19 m$

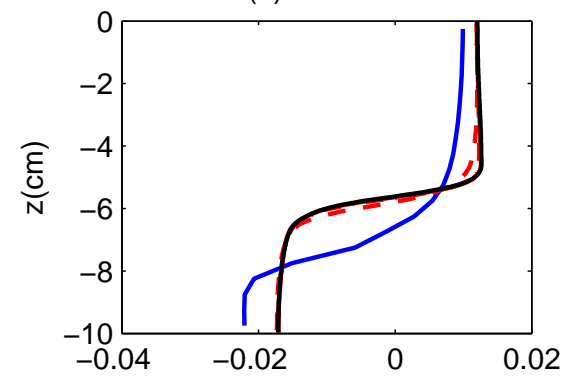

(c) $x=0.31 \mathrm{~m}$

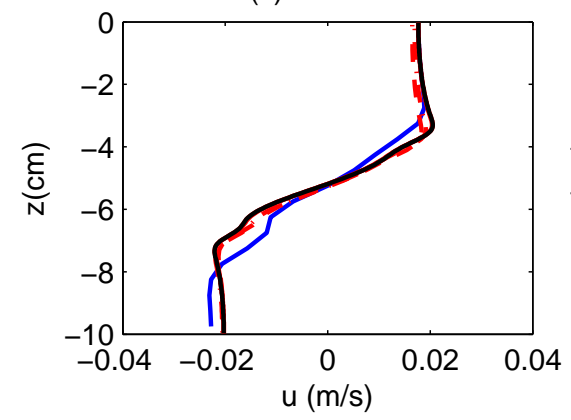

(b) $x=0.19 m$

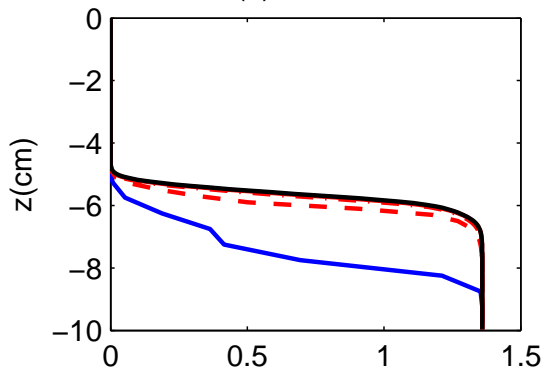

(d) $x=0.31 \mathrm{~m}$

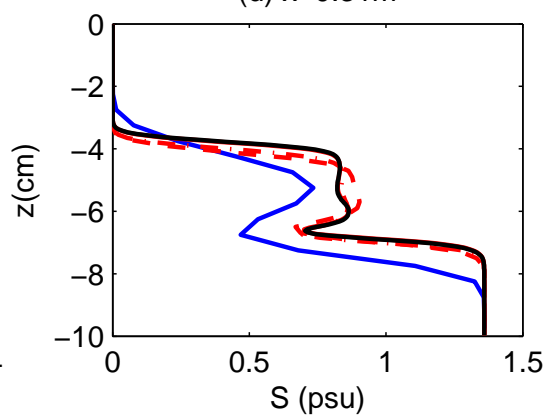

Figure 5: Comparisons of $u$ and salinity distributions at $t=20 \mathrm{~s}$ (Blue lines: $(20,20)$ layers; Black lines: (200,200) layers; Red-solid lines: (200,20) layers; Dash-dotted lines $(100,20)$ layers; Dashed lines: $(50,20)$ layers $)$. 
(a) $x=0.19 \mathrm{~m}$

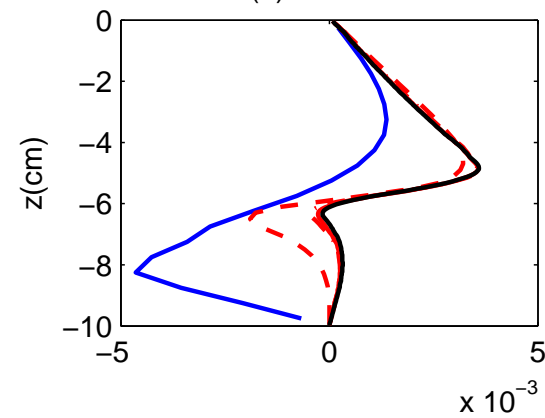

(c) $x=0.31 \mathrm{~m}$

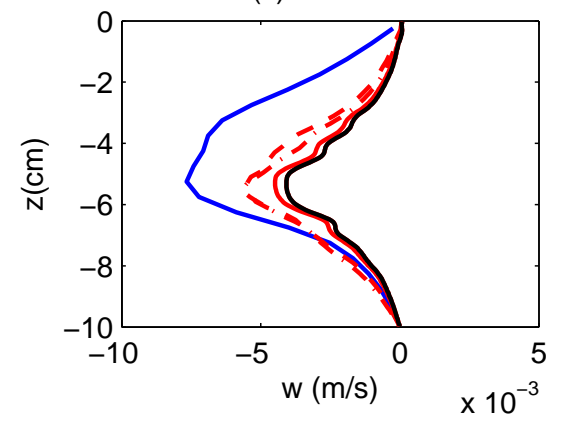

(b) $x=0.19 m$

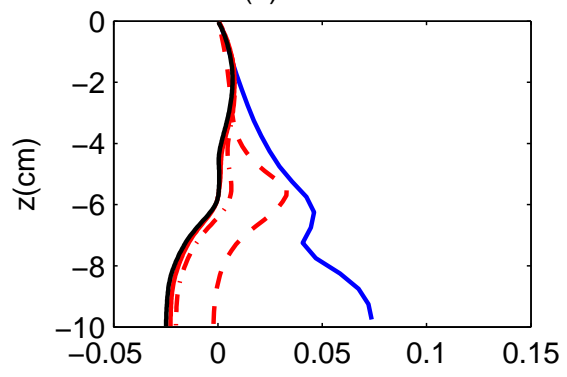

(d) $x=0.31 \mathrm{~m}$

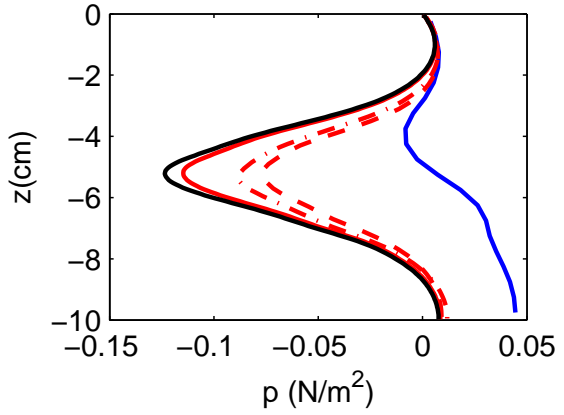

Figure 6: Comparisons of $w$ and dynamic pressure distributions at $t=20 \mathrm{~s}$ (Blue lines: $(20,20)$ layers; Black lines: $(200,200)$ layers; Red-solid lines: $(200,20)$ layers; Dash-dotted lines $(100,20)$ layers; Dashed lines: $(50,20)$ layers $)$. 

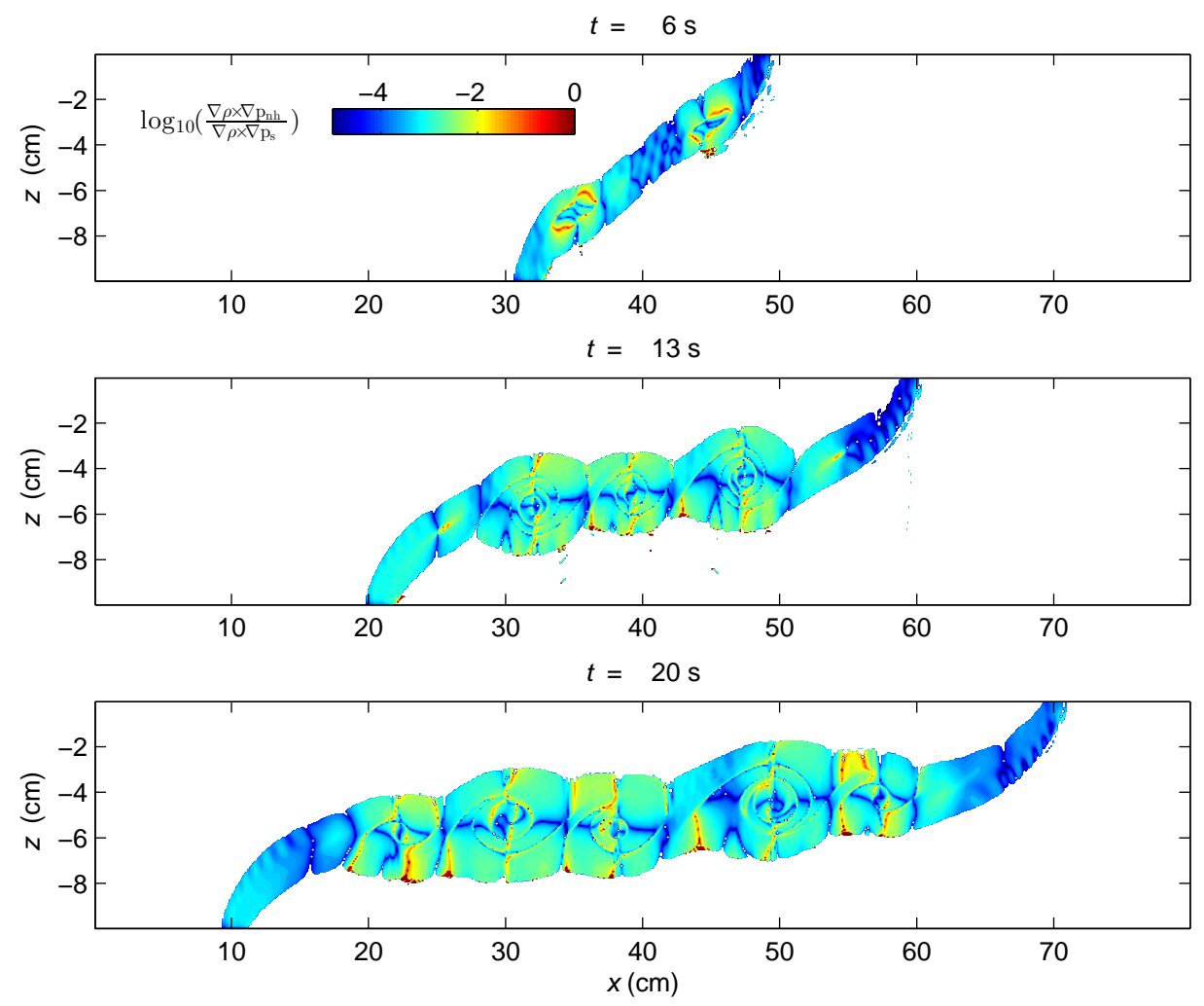

Figure 7: Snapshots of the ratio of the dynamic pressure production to the static pressure production at $t=6,13$ and $20 \mathrm{~s}$. 


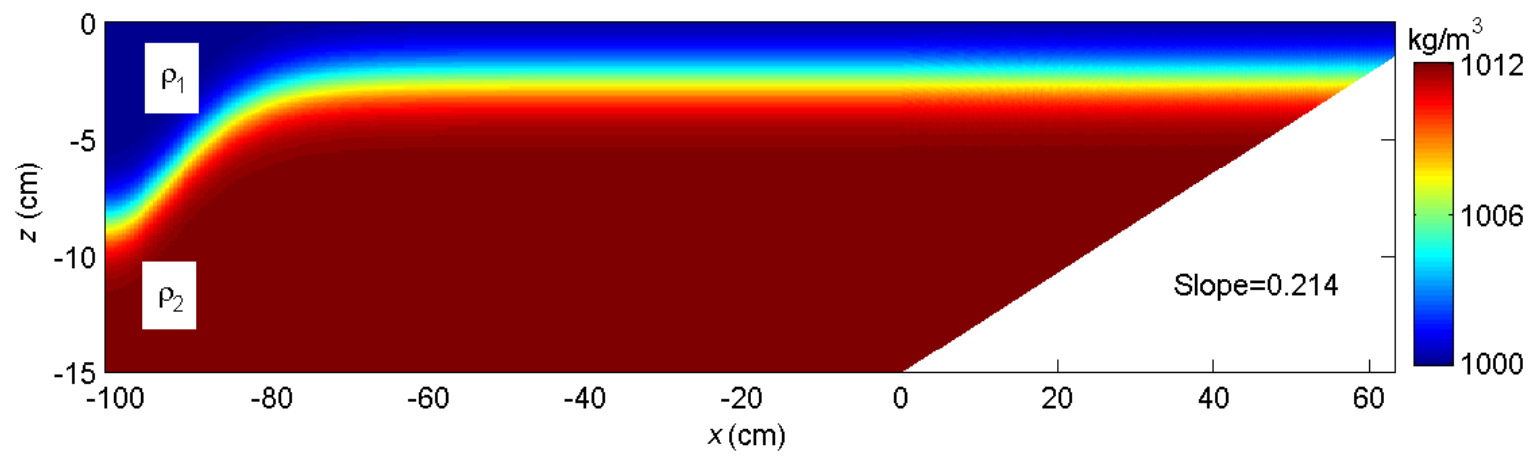

Figure 8: Initial density distribution of the internal wave breaking case. 

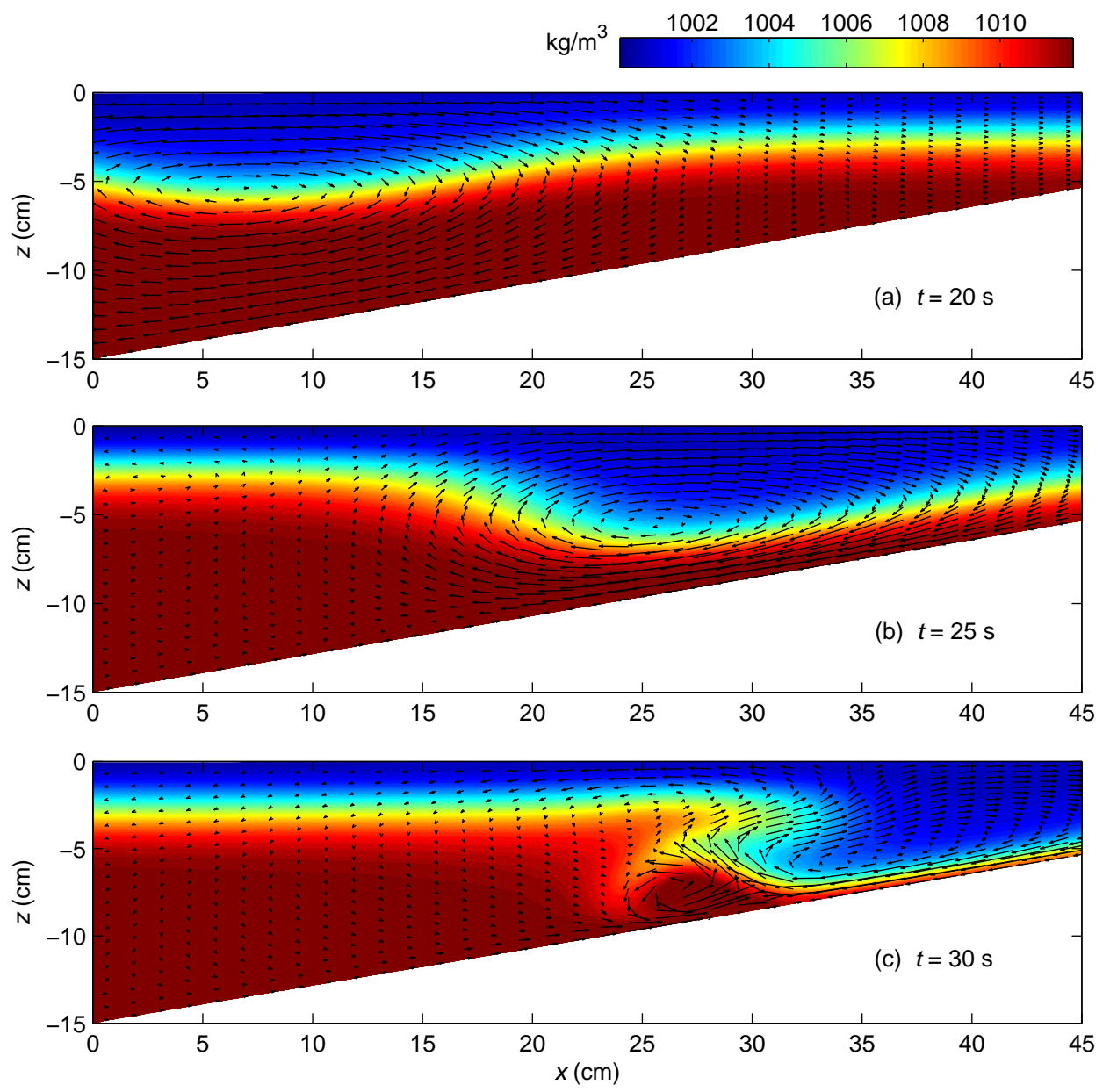

Figure 9: Snapshots of simulated density and velocity fields with $(200,200)$ layers. 

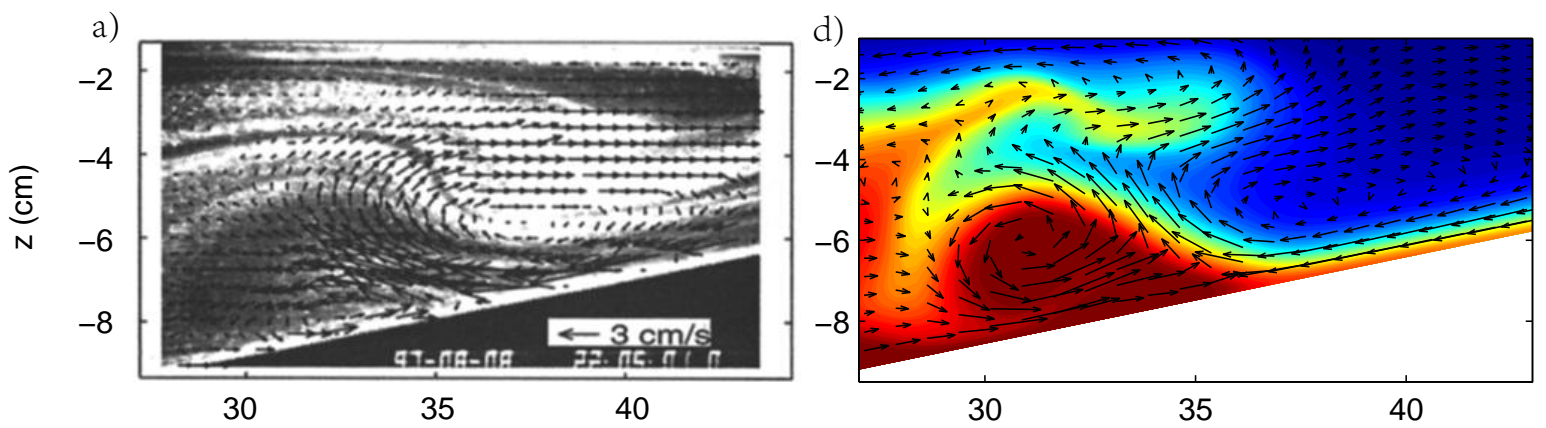

b)

e)
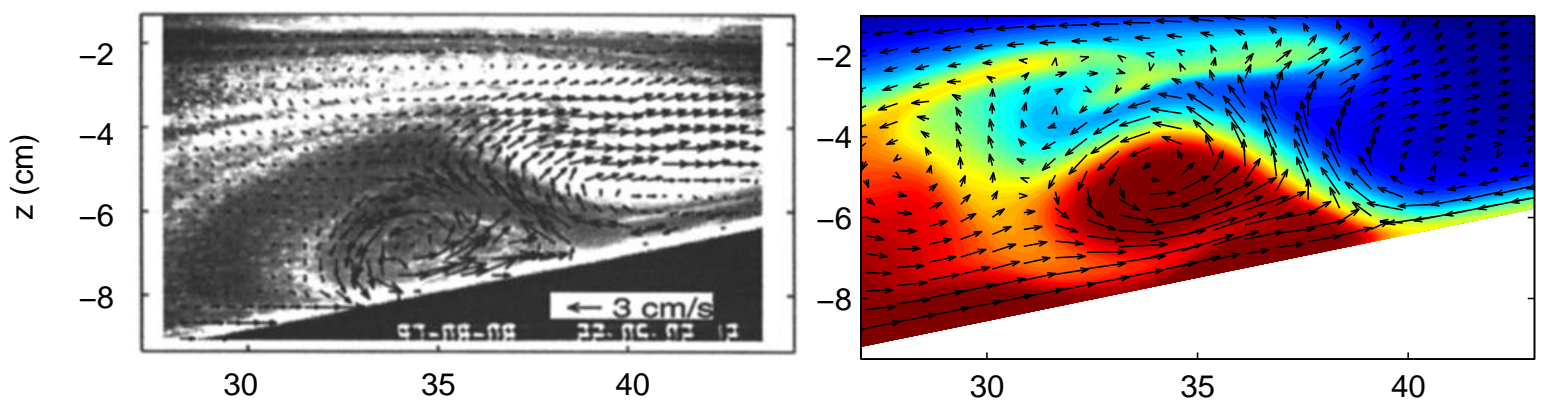

c)

f)
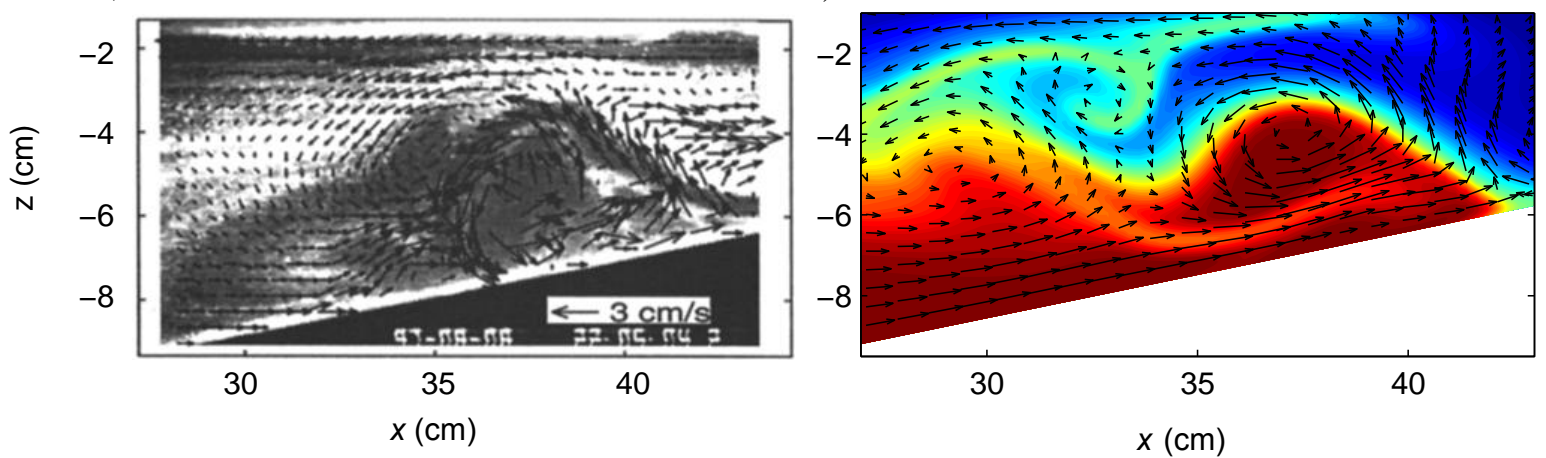

Figure 10: Comparisons of velocity field and density distributions between the laboratory observation(left, Michallet and Ivey 1999) and the (200,200) layers case (right). 

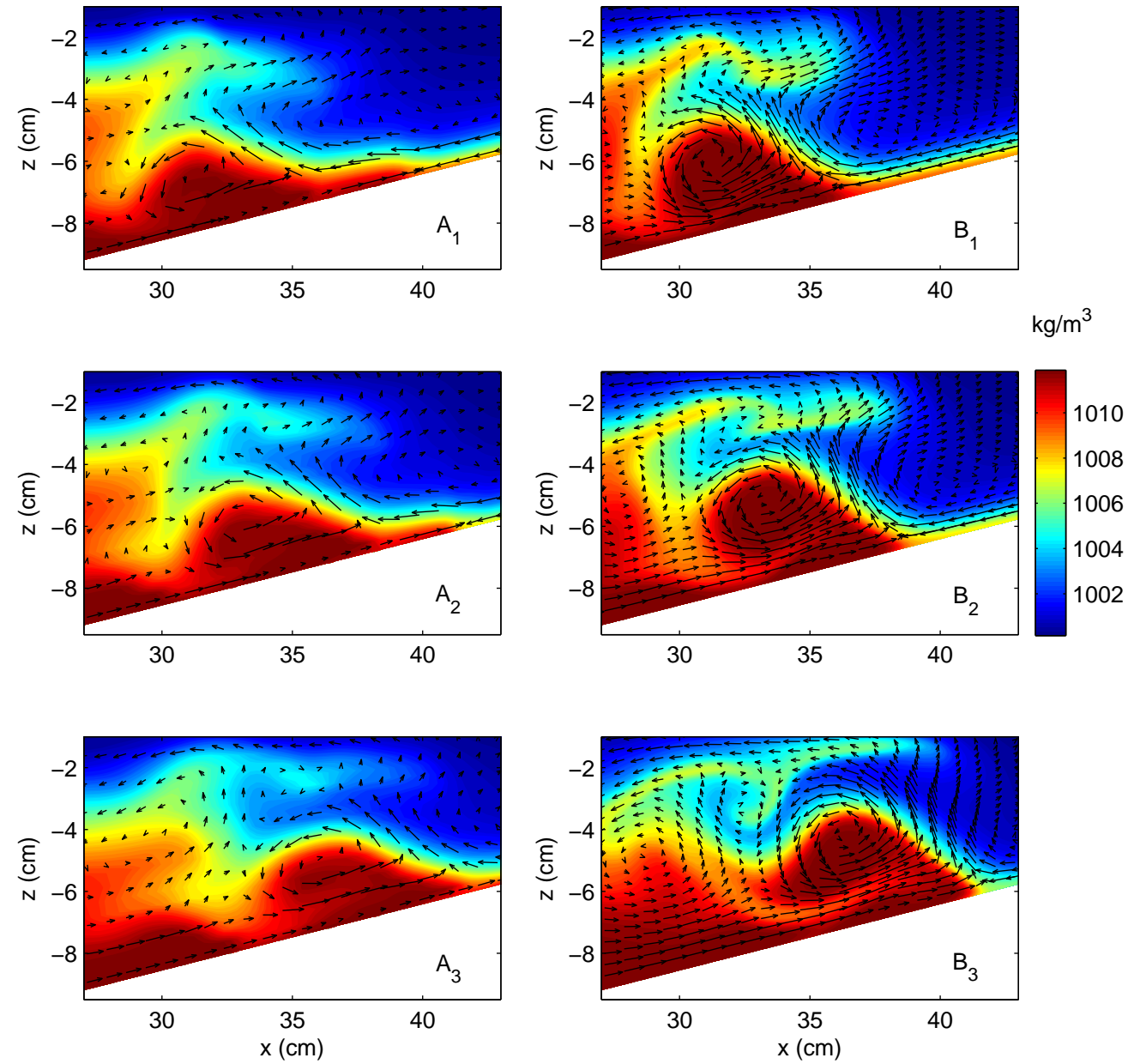

Figure 11: Velocity and density distributions for cases of $(20,20)$ layers $\left(A_{1}, A_{2}, A_{3}\right)$; $(200,20)$ layers $\left(B_{1}, B_{2}, B_{3}\right)$. 
(a) $x=33 \mathrm{~cm}$

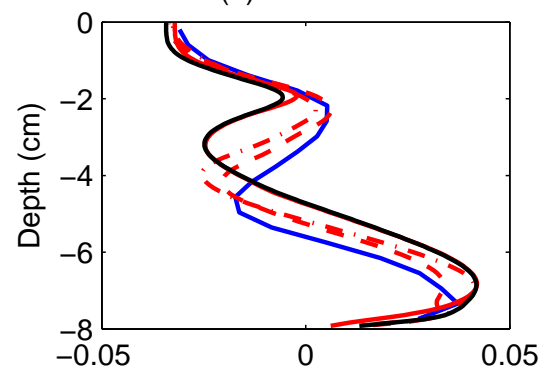

(c) $x=40 \mathrm{~cm}$

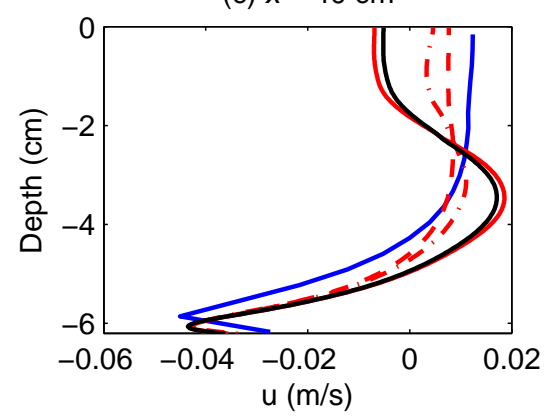

(b) $x=33 \mathrm{~cm}$

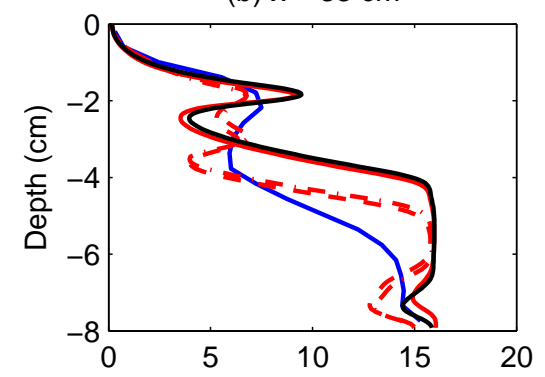

(d) $x=40 \mathrm{~cm}$

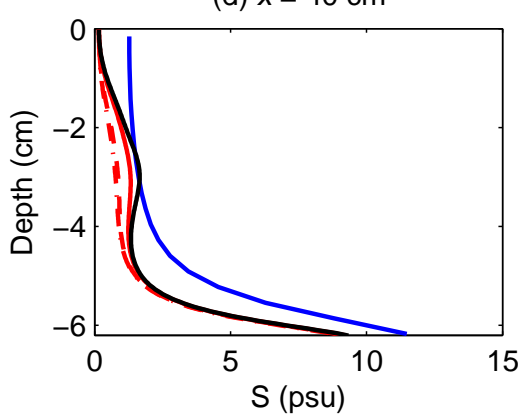

Figure 12: Comparisons of $u$ and salinity distributions at $t=33.5 \mathrm{~s}$ (Blue lines: $(20,20)$ layers; Black lines: $(200,200)$ layers; Red-solid lines: $(200,20)$ layers; Dash-dotted lines $(100,20)$ layers; Dashed lines: $(50,20)$ layers. 
(a) $x=33 \mathrm{~cm}$

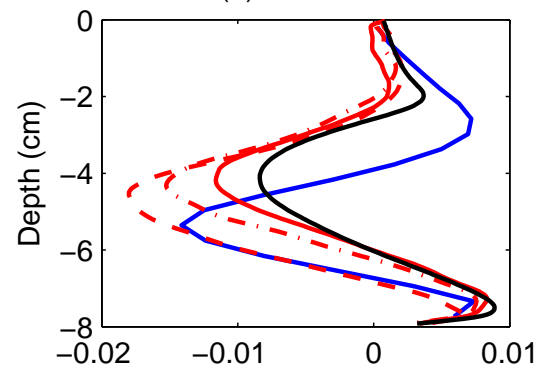

(c) $x=40 \mathrm{~cm}$

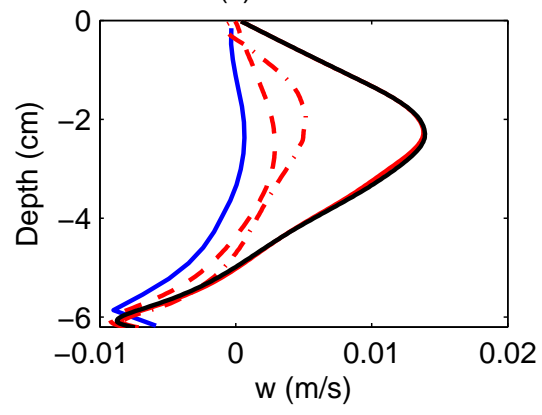

(b) $x=33 \mathrm{~cm}$

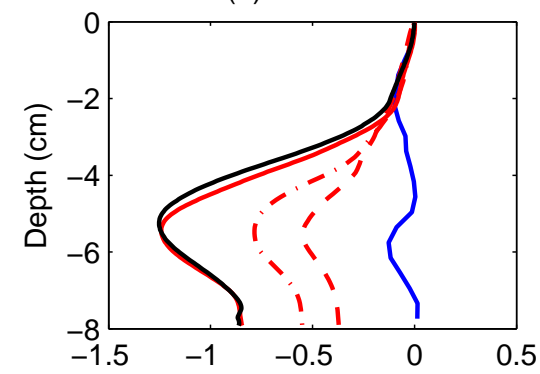

(d) $x=40 \mathrm{~cm}$

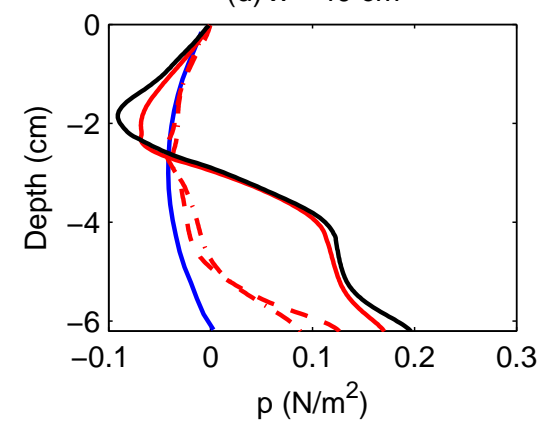

Figure 13: Comparisons of $w$ and dynamic pressure distributions at $t=33.5 \mathrm{~s}$ (Blue lines: $(20,20)$ layers; Black lines: (200,200) layers; Red-solid lines: $(200,20)$ layers; Dash-dotted lines $(100,20)$ layers; Dashed lines: $(50,20)$ layers.

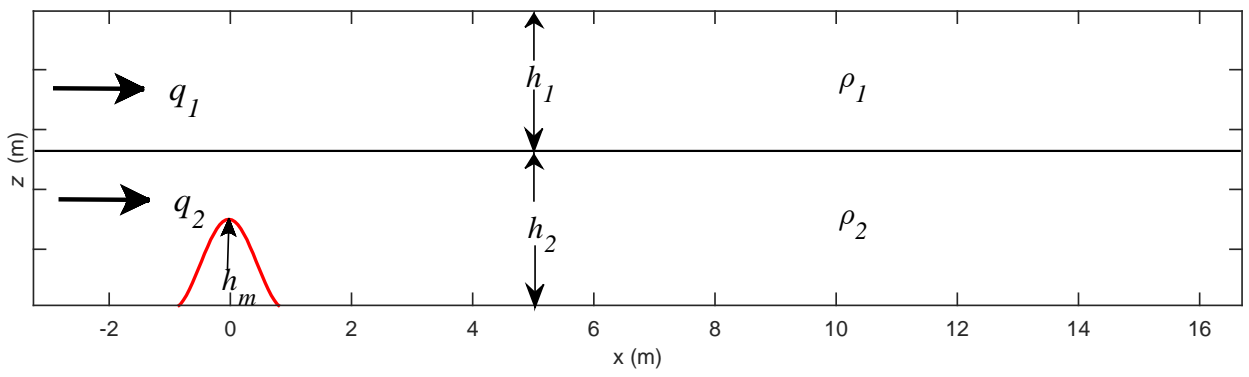

Figure 14: A schematic for the initial conditions in the case of the internal hydraulic jump. 


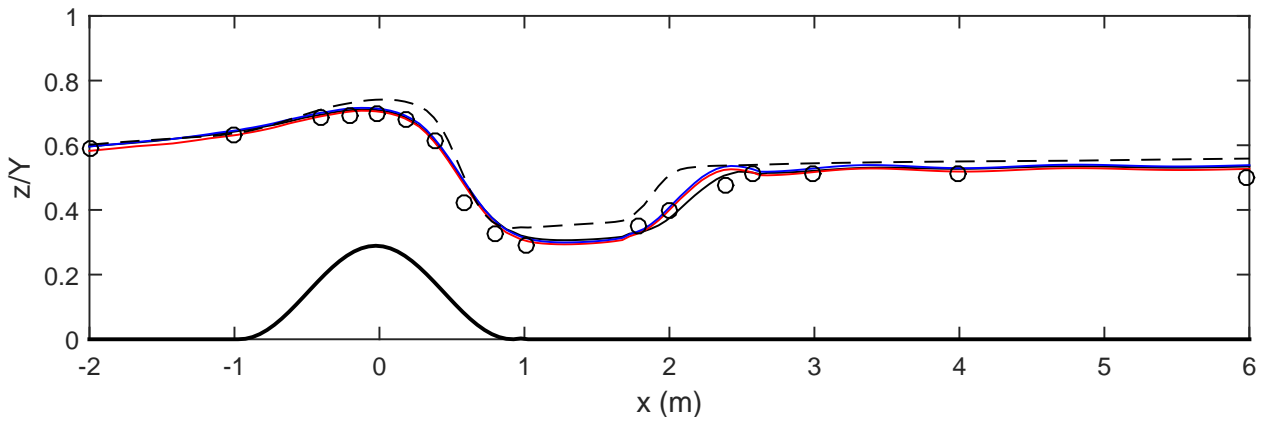

Figure 15: Comparisons of the interface positions (Black line: $(40,40)$ layers; Red line: $(40,20)$ layers; Blue line: $(40,10)$ layers; Dashed line: hydrostatic prediction; Circles: laboratory data conducted by Lawrence [1993], $Y=h(0)+\eta(x)$ is the free surface position, $h(0)$ is the depth at the west boundary). 

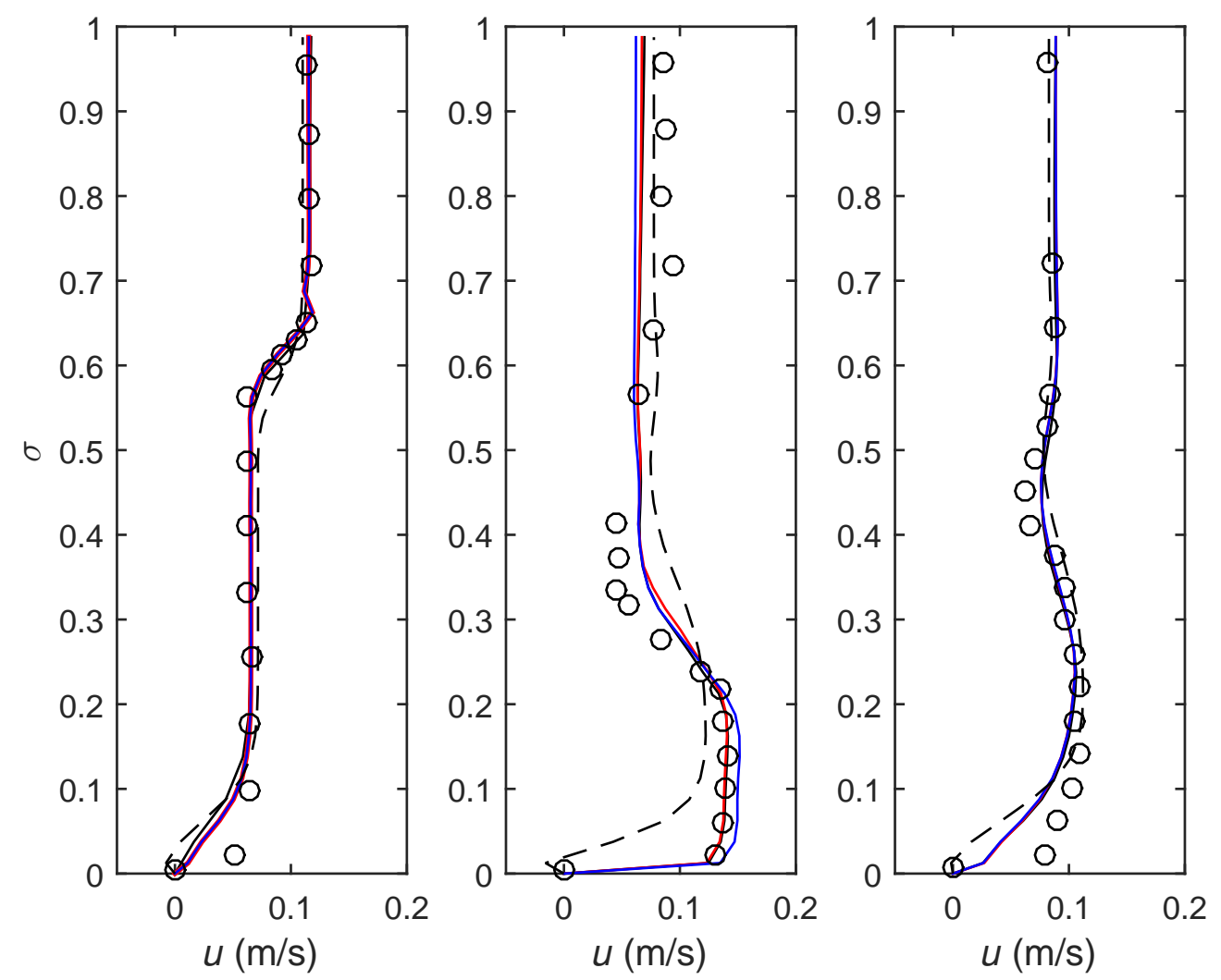

Figure 16: Comparisons of the $u$ profiles at $x=-1.0 \mathrm{~m}, 1.0 \mathrm{~m}$, and $5.0 \mathrm{~m}$ (from left to right) Black lines: $(40,40)$ layers; Red lines: $(40,20)$ layers; Blue lines: $(40,10)$ layers; Dashed lines: Hydrostatic predictions; Circles: laboratory data conducted by Lawrence [1993]. 

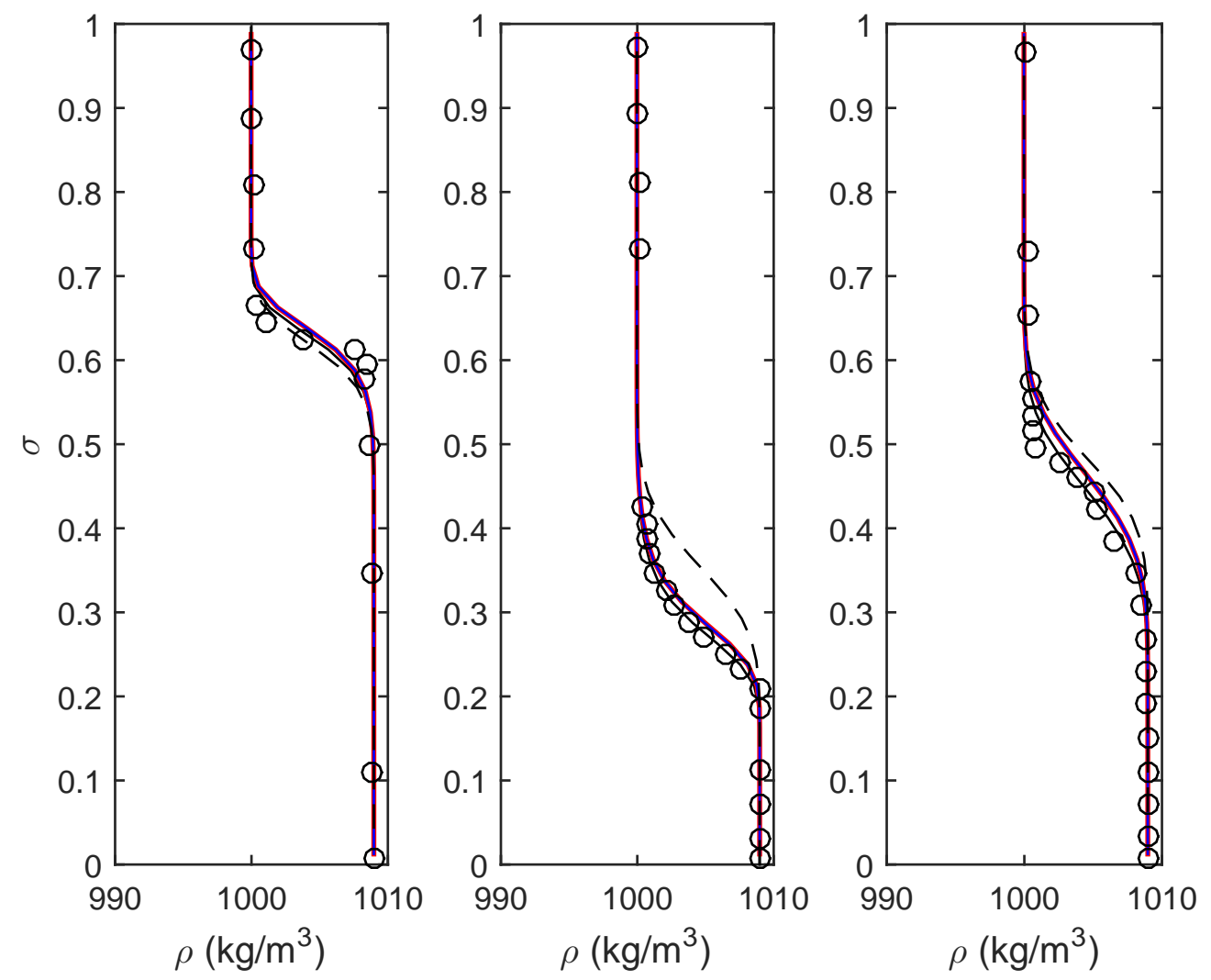

Figure 17: Comparisons of the density profile at $x=-1.0 \mathrm{~m}, 1.0 \mathrm{~m}$, and $5.0 \mathrm{~m}$ (from left to right) Black lines: $(40,40)$ layers; Red lines: $(40,20)$ layers; Blue lines: $(40,10)$ layers; Dashed lines: Hydrostatic predictions; Circles: laboratory data conducted by Lawrence [1993]. 


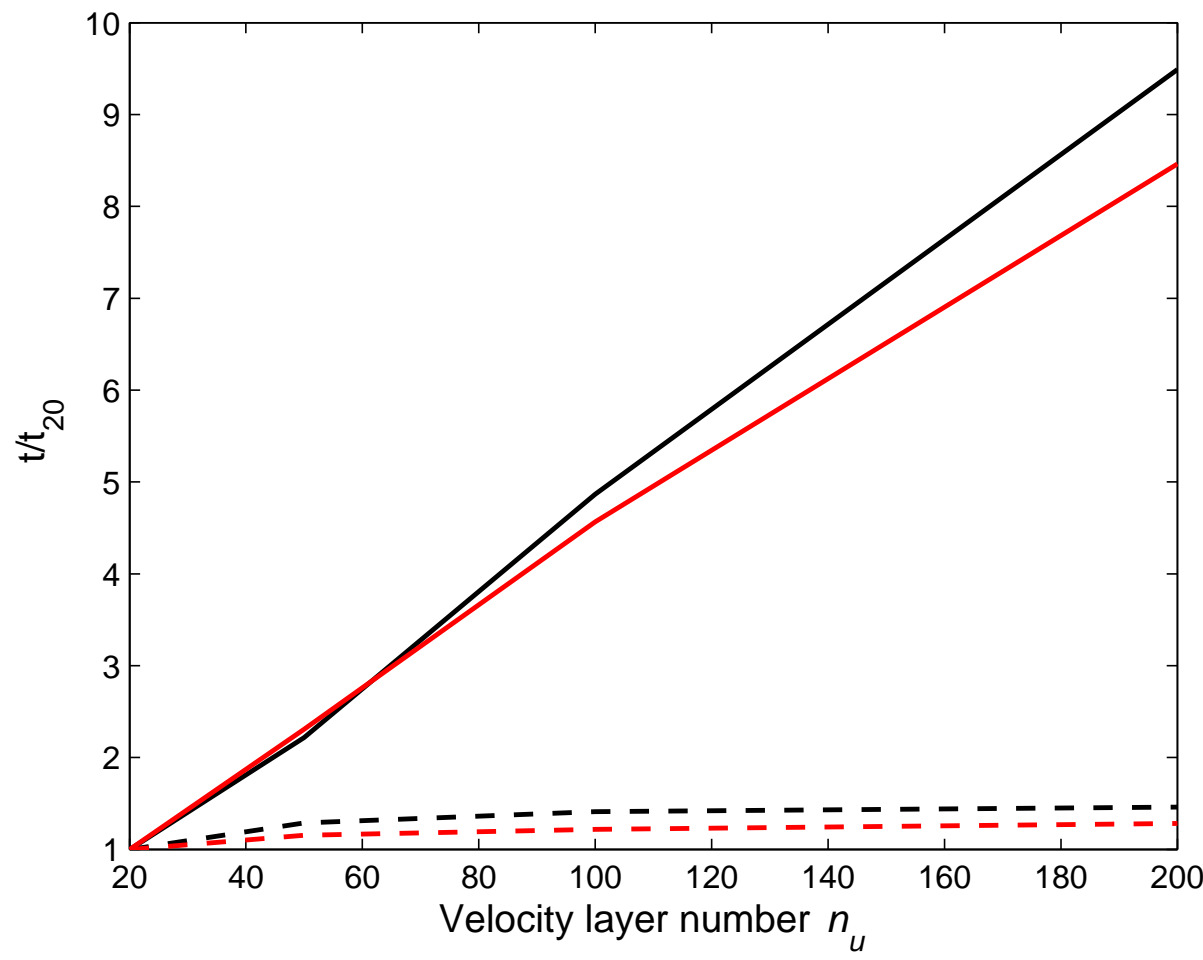

Figure 18: Computational time ratio for solving Poisson equation and interpolation. $t_{20}$ is the computational time for solving Poisson equation for the $(20,20)$ layers case. Black lines: the lock exchange case; Red lines: the internal wave case; Solid lines: the cases using the same numbers of pressure layers and velocity layers; Dashed lines: the cases with the number of $n_{p}=20$. 


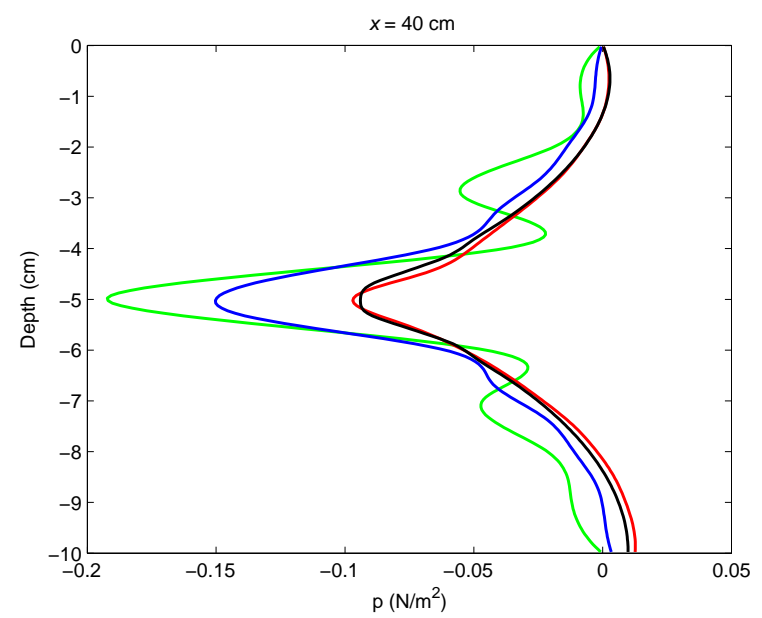

Figure 19: Comparisons of dynamic pressure distributions of the lock exchange case at $t=5$ s. Black line: $(200,200)$ layers; Red line: $(200,20)$ layers; Blue line: $(200,15)$ layers; Green line: $(200,10)$ layers

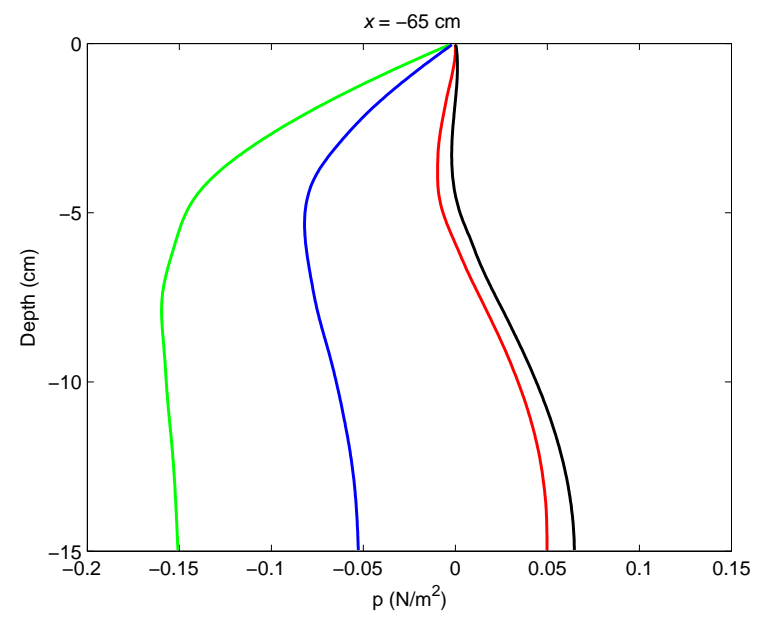

Figure 20: Comparisons of dynamic pressure distributions of the internal wave case at $t=5 \mathrm{~s}$. Black line: $(200,200)$ layers; Red line: $(200,20)$ layers; Blue line: $(200,15)$ layers; Green line: $(200,10)$ layers 


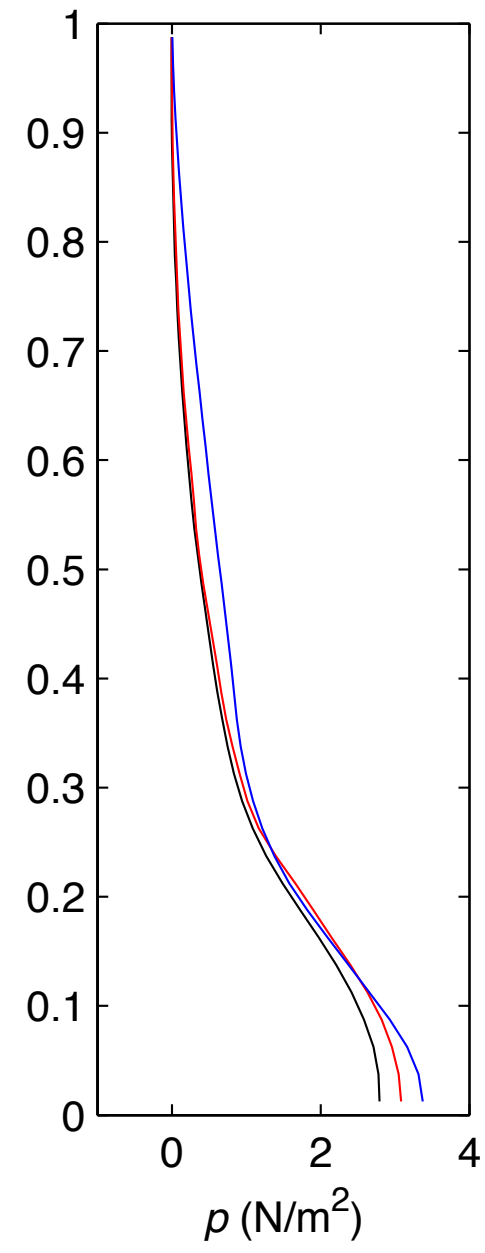

Figure 21: Comparisons of dynamic pressure distributions of the third case at $x=1.0 \mathrm{~m}$. Black line: $(40,40)$ layers; Red line: $(40,20)$ layers; Blue line: $(40,10)$ layers 